ARTICLE

https://doi.org/10.1038/s41467-019-12325-z

\title{
Tet inactivation disrupts YY1 binding and long-range chromatin interactions during embryonic heart development
}

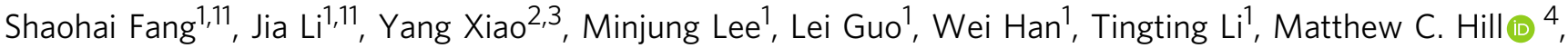 \\ Tingting Hong ${ }^{1}$, William Mo ${ }^{1}$, Rang $\mathrm{Xu}^{5}$, Ping Zhang ${ }^{5}$, Fen Wang ${ }^{6}$, Jiang Chang ${ }^{6}$, Yubin Zhou (1) 6,7, \\ Deqiang Sun (1) ${ }^{1}$, James F. Martin ${ }^{2,3,4,8}$ \& Yun Huang (i) 1,9,10
}

Tet-mediated DNA demethylation plays an important role in shaping the epigenetic landscape and chromatin accessibility to control gene expression. While several studies demonstrated pivotal roles of Tet in regulating embryonic development, little is known about their functions in heart development. Here we analyze DNA methylation and hydroxymethylation dynamics during early cardiac development in both human and mice. We find that cardiac-specific deletion of Tet2 and Tet3 in mice (Tet2/3-DKO) leads to ventricular non-compaction cardiomyopathy (NCC) with embryonic lethality. Single-cell RNA-seq analyses reveal a reduction in cardiomyocyte numbers and transcriptional reprogramming in cardiac tissues upon Tet2/3 depletion. Impaired DNA demethylation and reduced chromatin accessibility in Tet2/3-DKO mice further compromised Ying-yang1 (YY1) binding to its genomic targets, and perturbed high-order chromatin organization at key genes involved in heart development. Our studies provide evidence of the physiological role of Tet in regulating DNA methylation dynamics and chromatin organization during early heart development.

\footnotetext{
${ }^{1}$ Center for Epigenetics \& Disease Prevention, Institute of Biosciences and Technology, Texas A\&M University, Houston, TX 77030, USA. ${ }^{2}$ Texas Heart Institute, Cardiomyocyte Renewal Lab, Houston, TX 77030, USA. ${ }^{3}$ Department of Molecular Physiology and Biophysics, Baylor College of Medicine, One Baylor Plaza, Houston, TX 77030, USA. ${ }^{4}$ Program in Developmental Biology, Baylor College of Medicine, One Baylor Plaza, Houston, TX 77030, USA. ${ }^{5}$ Xinhua Hospital, School of Medicine, Shanghai Jiao Tong University, Shanghai, China. ${ }^{6}$ Center for Translational Cancer Research, Institute of Biosciences and Technology, Texas A\&M University, Houston, TX 77030, USA. ${ }^{7}$ Department of Medical Physiology, College of Medicine, Texas A\&M University, College Station, TX 77843, USA. ${ }^{8}$ Cardiovascular Research Institute, Baylor College of Medicine, Houston, TX 77030, USA. ${ }^{9}$ CPRIT Scholar in Cancer Research, Houston, USA. ${ }^{10}$ Department of Molecular \& Cellular Medicine, College of Medicine, Texas A\&M University, College Station, TX 77843, USA. ${ }^{11}$ These authors contributed equally: Shaohai Fang, Jia Li. Correspondence and requests for materials should be addressed to

D.S. (email: dsun@tamu.edu) or to J.F.M. (email: jfmartin@bcm.edu) or to Y.H. (email: yun.huang@tamu.edu)
} 
T he Ten-Eleven Translocation (TET) protein-mediated DNA modification pathway plays an important role in regulating DNA methylation and demethylation homeostasis during development ${ }^{1-3}$. Deletion of all three TET genes (TET1-3) impairs differentiation of both human and mouse embryonic stem cells $(\mathrm{ESCs})^{4,5}$. Furthermore, Tet-triple deficient mice exhibits developmental defects at the gastrulation stage ${ }^{2}$, indicating an indispensable role of Tet enzymes in early embryonic development. TET belongs to the $\mathrm{Fe}^{2+}$ and 2-oxoglutarate-dependent dioxygenase family that successively oxidizes 5 -methylcytosine $(5 \mathrm{mC})$ to 5-hydroxymethylcytosine $(5 \mathrm{hmC}), 5$-formylcytosine $(5 \mathrm{fC})$ and 5 -carboxylcytosine $(5 \mathrm{caC})^{6-9} .5 \mathrm{hmC}$ is one of the most abundant and relatively stable modifications among all these oxidized forms of DNA methylation ${ }^{7,8}$. TET-catalyzed DNA hydroxymethylation is enriched at enhancer and open chromatin regions during cellular differentiation and embryonic development ${ }^{10-13}$, thereby pointing to yet-to-be-clarified functions of Tet $/ 5 \mathrm{hmC}$ in chromatin biology and gene regulation. Somatic mutations of TET2 are frequently detected in individuals with clonal hematopoiesis, which are closely associated with high risk of cardiovascular disease $^{14-16}$.

Cardiac differentiation during embryonic development is tightly regulated through precise control over gene expression, when cells receive a multitude of intracellular and extracellular cues $^{17}$. Epigenetic factors, such as DNA modifying enzymes DNMTs, play indispensable roles in choreographing this exquisitely coordinated process by directly participating in the programming of cardiac transcriptional networks, thereby exerting control over gene expression to orchestrate early heart development ${ }^{18-20}$. Aberrant epigenetic modifications arising from genetic alterations in these key enzymes and/or environmental risk factors, such as folate deficiency, may cause developmental defects in the heart and potentially lead to embryonic lethality in mice, as well as human cardiomyopathies ${ }^{21-23}$. A deeper understanding of epigenetic regulatory mechanisms that modulate cardiac gene expression is crucial for deciphering the molecular etiology of congenital heart defects.

In this study, we systematically investigated the DNA methylation and hydroxymethylation dynamics during early cardiac development in both human and mice. We generated a cardiacspecific Tet 2 and Tet 3 double deficient mouse model to investigate the function of Tet-mediated DNA modifications during early cardiac development. These mice developed noncompaction cardiomyopathy (NCC) with severe developmental defects in the ventricular wall. With this disease-relevant in vivo model, we further unveiled previously-unrecognized roles of Tetmediated DNA hydroxymethylation in regulating chromatin accessibility to facilitate the genomic recruitment of one key transcription factor, Ying-Yang 1 (YY1). Excitingly, our study uncovered a crucial role of Tet $/ 5 \mathrm{hmC}$ in modulating long-range chromatin interactions to coordinate higher-order chromatin organization during embryonic heart development.

\section{Results}

Dynamic $5 \mathrm{mC}$ and $5 \mathrm{hmC}$ changes during heart development. To evaluate DNA methylation dynamics during mammalian heart development, we performed whole-genome bisulfite sequencing (WGBS; for $5 \mathrm{mC}$ profiling) and CMS-IP-seq (for $5 \mathrm{hmC}$ profiling) in both human and mouse embryonic heart tissues (Supplementary Table 1). For human heart tissues, we analyzed DNA methylation and hydroxymethylation at the Carnegie Stage (CS) 13 and 14, which are analogous to embryonic day 9.5 (E9.5) to E10.5 of the murine heart developmental stages $^{24}$. For mouse hearts, we analyzed DNA methylation dynamics using WGBS data available in ENCODE ${ }^{25}$ at different embryonic developmental stages (E11.5, 12.5, 13.5, 14.5, 15.5, 16.5 , P0). We also performed CMS-IP-seq using mouse embryonic cardiac tissues to compare $5 \mathrm{hmC}$ levels at the E12.5 stage.

We first comprehensively analyzed the DNA methylation dynamics in mouse hearts since the ENCODE data covered most of the key cardiac developmental stages. Although the global DNA methylation levels remained stable during embryonic development, ranging from 0.736 to 0.755 across all the stages (Fig. 1a), we were still able to locate 21,467 differentially methylated regions (DMRs, defined as $>20 \%$ methylation change, FDR $\leq 0.05)$ that covered $105,710 \mathrm{CpG}$ sites from E11.5 to P0 (Fig. 1b, Supplementary Fig. 1A), revealing dynamic changes in focal rather than global DNA methylation during cardiac development. Notably, very few DMRs (1\%) were commonly shared among the analyzed developmental stages (Supplementary Fig. 1B), and the majority of DMRs ( 99\%) were identified at different genomic regions for each developmental stage. This finding suggests that the observed focal DNA methylation changes are stage-specific, rather than occurring at the same genomic regions, during embryonic heart development. DNA Genomic Regions Enrichment of Annotations Tool (GREAT) ${ }^{26}$ analysis on all the identified DMRs further revealed that these regions are enriched at the cis-regulatory elements of genes essential for embryonic development and cardiac function, such as Bmp10 and Tnnt2 (Fig. 1c, d). Among all identified DMRs between adjacent developmental stages, approximately 66\% $(14,155$ out of 21,476 DMRs) exhibited a reduction in DNA methylation when progressing to the next developmental stage (defined as hypoDMRs; Fig. 1b). Notably, more than $80 \%$ of DMRs were classified as hypoDMRs during the E12.5-to-E13.5 or E16.5-to-P0 transitions (Fig. 1b), suggesting a pronounced reduction in local DNA methylation possibly through DNA demethylation in these development stages.

Since Tet-mediated DNA methylation oxidation, particularly DNA hydroxymethylation, is a key intermediate step for DNA demethylation during development, we next measured the global changes of $5 \mathrm{hmC}$ by a dot-blot assay ${ }^{27,28}$ in murine heart tissues collected at different developmental stages (E12.5, 14.5, 16.5, 18.5 and P0; Fig. 1e) $)^{13,29}$. We observed a gradual increase of $5 \mathrm{hmC}$ during heart development (Fig. 1e), suggesting that Tet-mediated DNA hydroxymethylation regulates DNA methylation dynamics during heart development. Real-time quantitative PCR (qPCR) also unveiled dynamic changes in Tet expression in cardiac tissues isolated at these developmental stages (Supplementary Fig. 1C). Immunohistochemistry (IHC) staining in E12.5 heart tissues revealed strong signals for $5 \mathrm{mC}, 5 \mathrm{hmC}, 5 \mathrm{fC}$, and to a lesser extent, for $5 \mathrm{caC}$ (Supplementary Fig. 1D). In parallel, IHC analysis of Tet1 and Tet2 at the same developmental stage (E12.5) confirmed strong Tet1 expression in myocardium; while Tet2 expression was abundantly detected in all three layers of heart wall, including myocardium, epicardium and endocardium (Supplementary Fig. 1E). Similarly, we observed strong $5 \mathrm{hmC}$ signals in human embryonic heart tissues at CS12 and 14 (Supplementary Fig. 1F), as well as strong IHC signals for TET1 and TET2 staining across heart tissues (Supplementary Fig. 1G). Real-time $\mathrm{qPCR}$ analysis revealed dynamic changes in the expression of TET and DNMT family members in the early stages of human heart development (CS11-14) (Supplementary Fig. $1 \mathrm{H})$. The lack of Tet3 IHC analysis is due to the unavailability of a reliable Tet3 antibody tailored for IHC analysis.

To directly investigate $5 \mathrm{hmC}$ distribution, we performed CMSIP-seq for genome-wide $5 \mathrm{hmC}$ profiling on isolated E12.5 mouse embryonic hearts. We observed strong $5 \mathrm{hmC}$ enrichment at identified DMRs (Fig. 1b) during heart development, with higher enrichment of $5 \mathrm{hmC}$ at hypoDMRs than at hyperDMRs 


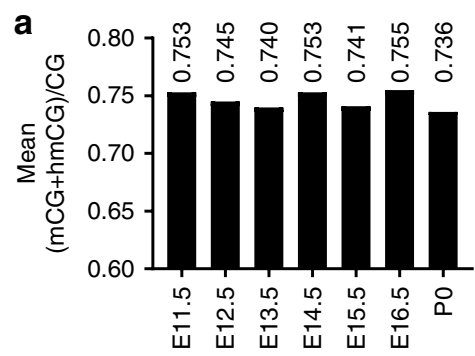

C

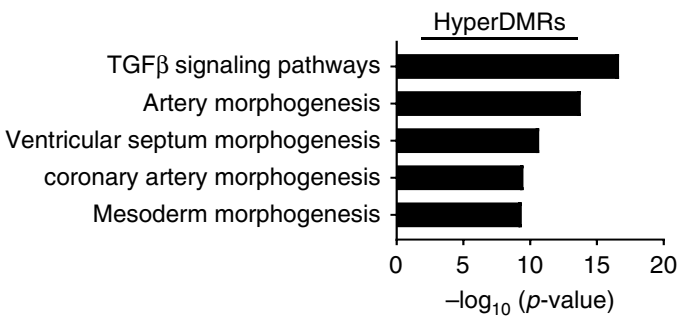

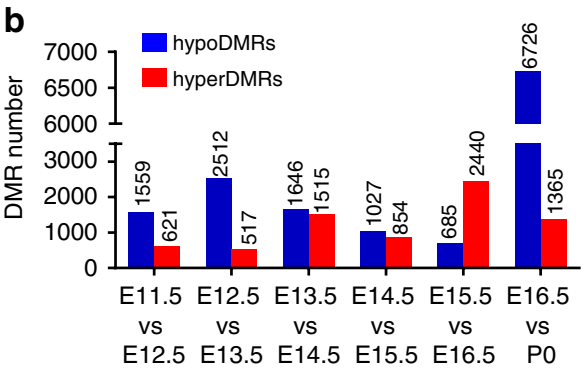

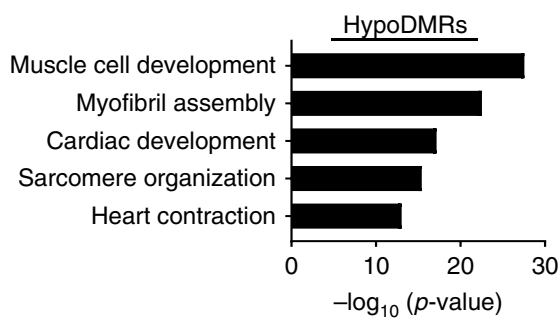

d

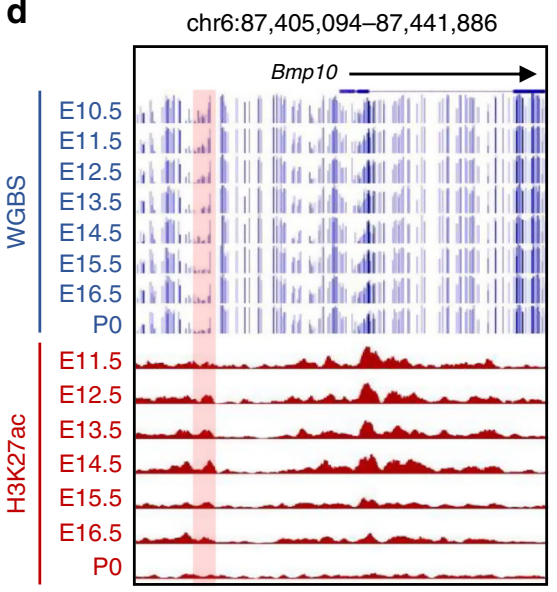

chr1:135,830,438-135,871,463

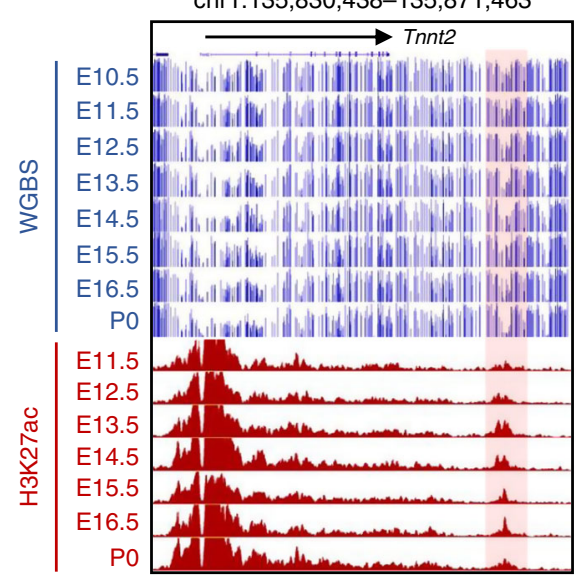

e 2-fold serial dilutions
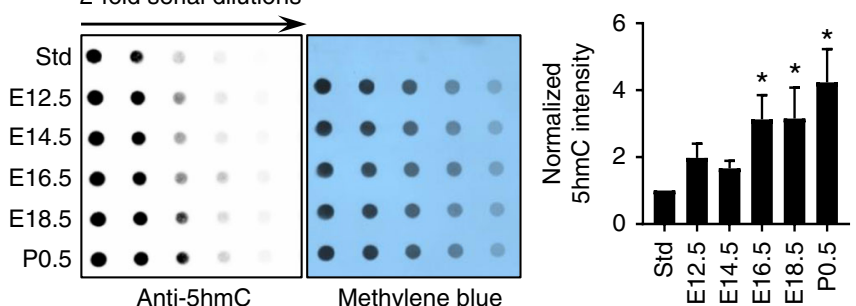

$\mathbf{f}$
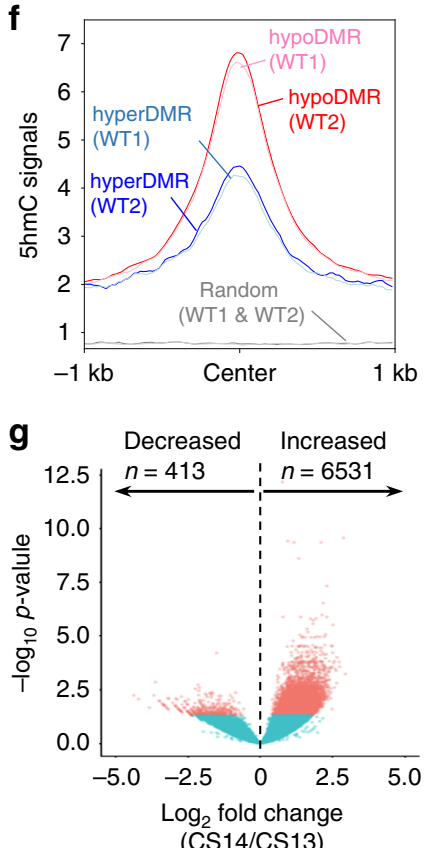

(CS14/CS13)
(hyperDMR defined as DMRs with significant increase of $5 \mathrm{mC}$ signal when transitioning into the next developmental stage; Fig. 1f). We further analyzed DNA methylation and $5 \mathrm{hmC}$ enrichment in human embryonic heart tissues at CS13 and 14. We noticed a pronounced increase of $5 \mathrm{hmC}$ during the CS13-toCS14 transition (Fig. 1g, Supplementary Fig. 2A), coinciding with the strong induction of TET2 and TET3 expression at CS14 (Supplementary Fig. 1F). GREAT analysis implied that the genomic regions displaying significant differential DNA hydroxymethylation (designated DHMRs for differential hydroxymethylated regions) between the CS13 an $\bar{d}$ CS14 stages are closely associated with human embryonic and cardiac development (Supplementary Fig. 2B). For example, we observed an increase of $5 \mathrm{hmC}$ at proximal regions to NOTCH and NKX2.5, two genes that are essential for normal heart development (Supplementary Fig. 2C). Consistent with murine DNA methylation analysis, we did not detect significant global DNA methylation changes between the CS13 to CS14 developmental 
Fig. 1 Dynamic changes of DNA methylation and hydroxymethylation during embryonic heart development in mouse and human. a Quantification of global average DNA methylation levels (quantified as mean ( $\mathrm{mCG}+\mathrm{hmCG}$ )/CG) during mouse heart development (E11.5 to PO) based on WGBS data from ENCODE. b Numbers of differentially methylated regions (DMRs) that show increased (defined as hyperDMRs) or reduced DNA methylation (hypoDMRs) when mouse embryonic hearts progress into the next developmental stage. c GREAT analysis on hyperDMRs and hypoDMRs shown in Fig. $1 \mathrm{~b}$ revealed representative terms associated with embryonic development and cardiac functions. Corrected binomial raw p-value were calculated. $\mathbf{d}$ Representative genome browser views illustrating the focal DNA methylation (WGBS; blue) and H3K27ac (red) dynamics at genomic regions surrounding Bmp10 and Tnnt2 during cardiac development in mouse embryos (E10.5 to PO). e (Left) Global 5hmC levels in mouse heart tissues collected at five embryonic stages (E12.5, 14.5, 16.5, 18.5, and PO) measured by the dot-blot assay. Methylene blue was used as loading control for the total DNA input. (Right) Quantification of dot-blot assay. Data were shown as mean \pm S.D; $n=3$ independent experiments. ${ }^{\star} p<0.05$, compared with E12.5 (two-tailed Student's $t$-test were used). f $5 \mathrm{hmC}$ enrichment signals of control E12.5 heart tissues within hypoDMRs (two repeated samples; red and pink) and hyperDMRs (two repeated samples; blue and cyan) identified from Fig. 1b. Random genomic regions (gray) were used as control. $\mathbf{g}$ The Volcano plot of differentially enriched 5hmC regions (DHMRs; CS13 vs CS14) in human embryonic hearts

stages of human heart (Supplementary Fig. 2D). We detected 324 and 182 hypo- and hyper-DMRs, respectively, in the CS13-toCS14 transition (Supplementary Fig. 2E), suggesting focal DNA methylation dynamics during human heart development. Furthermore, most genomic regions displaying increased DNA hydroxymethylation (hyperDHMRs) exhibited reduction in DNA methylation (Supplementary Fig. 2F), suggesting $5 \mathrm{hmC}$-mediated focal DNA demethylation during the CS13-to-CS14 transition. Taken together, our epigenomic analyses validated that focal $5 \mathrm{mC}$ changes, arising from altered DNA hydroxymethylation, are closely associated with murine and human heart development.

Cardiac Tet $2 / 3$ loss causes non-compaction cardiomyopathy. To elucidate the function of Tet-mediated DNA methylation oxidation during heart development, we generated a cardiac tissue-specific Tet-deficient mouse model. Earlier studies have shown that Tet 1 and Tet 2 individual knockout mice display no overt cardiac phenotypes ${ }^{30-34}$. Meanwhile, germline knockout of Tet 3 resulted in embryonic lethality, preventing systematic studies on cardiac development ${ }^{3}$. To circumvent these limitations, we first crossed mice bearing a conditional Tet $3^{\text {flox/flox }}$ allele ${ }^{1}$ with the cardiomyocyte $(\mathrm{CM})$ progenitor driver line, $\mathrm{Nkx} 2.5-\mathrm{Cre}^{35}$, to yield cardiac-specific deletion of Tet3. Next, we crossed Tet $3^{\text {flox/ }}$ flox;Nkx2.5-Cre mice with Tet2KO mice to disrupt both Tet 2 and Tet3 genes in CM progenitors (abbreviated as Tet2/3-DKO; Supplementary Fig. 3A, B). While the heterozygous mice are viable and showed no obvious cardiac phenotypes, homozygous mice were embryonically lethal (Fig. 2a). We collected embryos at developmental stages starting from E12.5 for further analyses. No appreciable morphological abnormalities were observed in Tet2/ 3-DKO embryos at E12.5 and E15.5, although some Tet2/3-DKO embryos had evidence of hemorrhage (Supplementary Fig. 3C). After histological analysis on embryonic hearts collected at E12.5, $13.5,14.5$ and 15.5 , we found that Tet2/3-DKO embryos displayed severe cardiac developmental defects, including ventricular septal defect (VSD) and double outlet right ventricle (DORV) (Supplementary Fig. 3D). Tet2/3-DKO hearts showed abnormal ventricular chamber development starting from E13.5 (Fig. 2b). Specifically, Tet2/3-DKO hearts had ventricular non-compaction cardiomyopathy (NCC) phenotype, as evidenced by significantly reduced ventricular wall thickness and increased trabecular areas compared to controls (Fig. 2b, Supplementary Fig. 3D, E).

Next, we performed real-time qPCR to examine the expression of Nppa and Hey2, previously known to be implicated in ventricular NCC, in Tet2/3-DKO hearts (Fig. 2c). We detected a significant decrease of $N p p a$ and Hey2 expression in Tet2/3-DKO, thus validating the NCC phenotype at the molecular level. Because ventricular NCC has been shown to arise from defects in CM proliferation and/or increased cellular apoptosis ${ }^{36}$, we further performed immunofluorescent (IF) staining with cellular proliferation and apoptotic markers in WT and Tet2/3-DKO E12.5 hearts. We observed a significant reduction in the staining signals for the cellular proliferation marker Ki67 (Fig. 2d). With regard to cleaved caspase-3 as apoptotic marker, we failed to detect meaningful signals in E12.5 control and Tet2/3-DKO heart tissue (Supplementary Fig. 3G). Together, these results clearly established the physiological roles of Tet 2 and Tet 3 in mediating ventricular chamber development.

Tet2/3 regulate cardiac-specific transcription. We next performed transcriptomic profiling, with RNA-seq, in cardiac tissues collected from E12.5 and E15.5 embryos (Supplementary Table 1, Supplementary Fig. 4A). We identified a total of 2,101 differentially expressed genes (DEGs), with 1,268 down-regulated and 833 up-regulated, respectively, in Tet $2 / 3$ DKO samples collected at E12.5 embryos compared with controls (Fig. 3a). In parallel, we identified 374 up-regulated and 440 down-regulated genes in E15.5 embryonic hearts (Fig. 3a). Gene ontology (GO) analysis on these DEGs revealed the involvement of key signaling pathways (e.g., Notch and Bmp related signaling) that are known to be crucial for ventricular chamber development (Fig. 3b, Supplementary Fig. 4B-D) $)^{37,38}$. Furthermore, key genes involved in CM development (such as Nppa, Tnnt2, and Myh6) showed altered expression in the Tet2/3-DKO group (Supplementary Fig. 4B-D). These unbiased transcriptomic and bioinformatic analysis data provided further evidence to support a critical role of Tet $2 / 3$ in embryonic heart development.

To further examine the function of these Tet2/3-regulated DEGs identified from RNA-seq analysis, we sorted DEGs implicated in cardiac development based on GO analysis (Fig. 3c, left). Then we analyzed the expression levels of these genes using ENCODE RNA-seq data collected from mouse embryonic hearts at different developmental stages (E10.5 to P0; Fig. 3c, right). Interestingly, we found that the expression levels of these genes underwent gradual changes during heart development, suggesting that these Tet2/3-regulated genes are tightly and temporally controlled at different embryonic stages. For example, the expression levels of $M y l 2$, Tnnt2 and Nppa gradually increased during normal heart development based on ENCODE RNA-seq data; however, the expression of these genes were significantly decreased in Tet2/3-DKO heart tissue (Supplementary Fig. 4E), suggesting that deletion of Tet proteins disrupts the precise transcriptional regulation of these key genes to impair cardiac development.

scRNA-seq analysis in cardiac-specific Tet2/3 deficient mice. The above RNA-seq analyses were performed in bulk embryonic heart tissues collected at E12.5 and E15.5 that contain multiple cell types, including myocardium, epicardium, endocardium, fibroblasts and other non-heart tissue cells (e.g., hematopoietic cells). To avoid potentially biased results due to changes in cell types upon Tet $2 / 3$ knockout, we carried out single-cell RNA-seq 
a

\begin{tabular}{|c|c|c|c|c|c|c|}
\hline \multirow{2}{*}{ Stage } & \multirow{2}{*}{$\begin{array}{l}\text { Total } \\
\text { embryos } \\
\text { number }\end{array}$} & \multicolumn{2}{|c|}{$\begin{array}{c}\text { Tet2 }^{+/} \text {Tet }^{\mathrm{fl} / \mathrm{fl} /} ; \mathrm{Nkx} 2.5-\mathrm{Cre} \\
\text { (Het) }\end{array}$} & \multicolumn{2}{|c|}{ Tet2 $^{-1-}$ Tet $^{\text {fl/fl| }} ;$ Nkx2.5-Cre (DKO) } & \multirow{2}{*}{$\begin{array}{l}\text { Chi- } \\
\text { Square }\end{array}$} \\
\hline & & $\begin{array}{l}\text { Expected } \\
(50 \%)\end{array}$ & Actual & $\begin{array}{l}\text { Expected } \\
(50 \%)\end{array}$ & Actual & \\
\hline E12.5 & 103 & 52 & $43(41.7 \%)$ & 52 & 60 (58.3\%) & 0.094 \\
\hline E13.5 & 23 & 12 & 14 (60.9\%) & 12 & $9(39.1 \%)$ & 0.297 \\
\hline E15.5 & 27 & 14 & 19 (70.4\%) & 14 & $8(29.6 \%)$ & $0.034^{*}$ \\
\hline E16.5 & 17 & 9 & 13 (76.5\%) & 9 & $4(23.5 \%)$ & $0.029^{*}$ \\
\hline P0.5 & 12 & 6 & $12(100 \%)$ & 6 & 0 & $0.001^{* *}$ \\
\hline
\end{tabular}

b
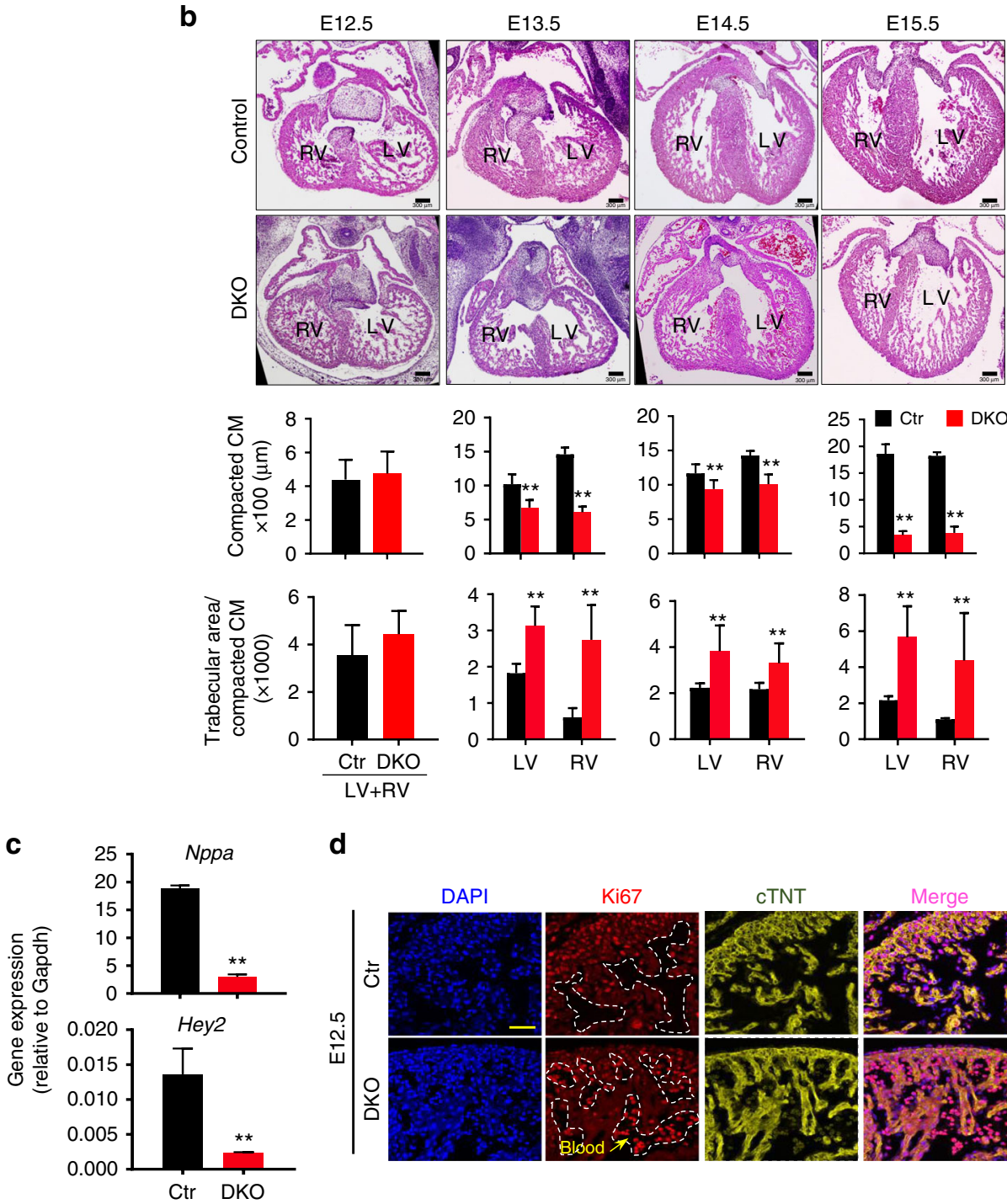

d
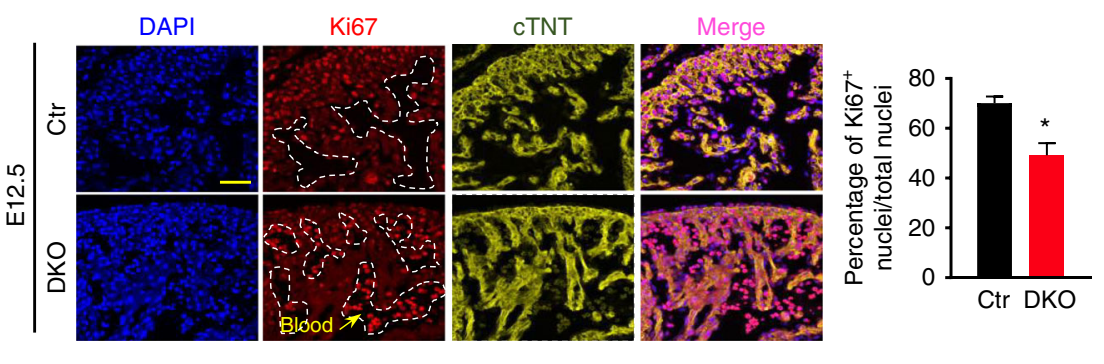

(scRNA-seq) on control and Tet2/3-DKO E12.5 and E15.5 hearts (Supplementary Table 1 and 3). Consistent with our histological analysis data (Fig. 2c), we observed minor differences in the cell types in E12.5 hearts between control and Tet2/3-DKO embryos. However, we noticed a massive reduction of the CMs (from $32.9 \%$ to $6.8 \%$ ) in the Tet2/3-DKO group at E15.5 (Fig. 3d, Supplementary Fig. 4F-G). Furthermore, consistent with our realtime qPCR analysis and bulk RNA-seq data described above (Figs $2 \mathrm{~d}$ and 3a), key genes involved in ventricular compaction, such as $\mathrm{Nppa}$ and $\mathrm{Hey2}$, were significantly down-regulated in E15.5 Tet2/3-DKO CMs when compared to controls (Fig. 3e). Furthermore, genes essential for cardiac development, such as Tbx20, Ttn and Gja1, were significantly down-regulated in Tet $2 /$ 3-DKO E15.5 CMs (Fig. 3e).

Since the CMs is mostly affected at the E15.5 stage, we then performed cluster analysis in E12.5 and E15.5 heart tissues from both control and Tet2/3-DKO mice using the MAGIC algorithm $^{39}$. In the CMs marked by Ttn, we found that Tet $2 / 3$ 
Fig. 2 Cardiac-specific deletion of Tet2 and Tet3 resulted in developmental defects in the ventricular chamber. a Genotyping analysis from Tet2-/-Tet3 $3^{f l o x /}$ flox and Tet2 ${ }^{+/-}$Tet3flox/flox; Nkx2.5-Cre interbreedings. ${ }^{\star} p<0.05,{ }^{* *} p<0.01$ (chi-squared test were used). b (Top) Representative H\&E staining images of embryonic heart tissues $(\times 4)$ collected at E12.5, 13.5, 14.5, and 15.5 stages from control and Tet2/3-DKO mice. (Bottom) Quantifications were performed by using the Image J software. Data were shown as mean \pm S.D; $n=36$ sections from 3 independent experiments. ${ }^{\star \star} p<0.01$ compared to control (twotailed Student's $t$-test were used). Scale bar: $300 \mu \mathrm{m}$. c Real-time qPCR to quantify the expression of Nppa and Hey2 in control and Tet2/3-DKO embryonic heart tissues collected at E12.5. Data were shown as mean \pm S.D; $n=3$ independent experiments. ${ }^{\star *} p<0.01$ (two-tailed Student's $t$-test were used). d (Left) Representative fluorescent imaging of embryonic heart tissues collected from E12.5 of control (top) or Tet2/3-DKO mice (bottom). DAPI (blue) was used for nuclear staining; Ki67 (red) was used as a proliferation marker; and cTnT (yellow) was used as the staining marker for cardiomyocytes. Cells demarcated within the white dashed lines are blood cells. (Right) Quantifications of the percentage of cardiomyocytes with positive Ki67 staining. Data were shown as mean \pm S.D; $n=4$ independent experiments (a total of 525 and 568 cardiomyocytes were quantified from control and Tet2/3-DKO embryos, respectively). ${ }^{\star} p<0.05$ compared to control (two-tailed Student's t-test were used). Scale bar: $50 \mu \mathrm{m}$

depletion led to substantial changes in the distribution patterns of principal components at E15.5 (Fig. 3f), but not at E12.5 (Supplementary Fig. 4H). Tet2/3-DKO CMs are clustered at Ttn-low expressed cells compared with control in E15.5 stage (Fig. 3f). Next, we performed further analyses on CMs based on the expression levels of two well-known cardiac chamber development-related genes $\mathrm{Tb} \times 2 \mathrm{O}$ and Hey2, and found a positive correlation between the expression levels of these two genes in normal E15.5 CMs (Fig. 3g), implying that the abundancy of the expression of Tbx20/Hey2 might be correlated with the maturation status of CMs. By contrast, E15.5 CMs collected from Tet2/3DKO embryos were all clustered into a prominent Tbx20lo${ }^{w} \mathrm{Hey}^{\text {low }} \mathrm{Ttn}^{\text {low }}$ population (Fig. 3g), suggesting that cardiacspecific Tet $2 / 3$ deletion might block CMs maturation during embryonic development. Together, these data indicated that Tet2 and Tet 3 are essential for regulating the expression of genes that are critical for ventricular maturation.

Global decrease of $5 \mathrm{hmC}$ in Tet2/3-deficient embryonic heart. We next monitored the global changes of $5 \mathrm{hmC}$ in E12.5 Tet2/3DKO heart tissues by immunofluorescent (IF) staining and the dot-blot assay (Fig. 4a, b). Both methods confirmed a substantial decrease of $5 \mathrm{hmC}$ in Tet $2 / 3-\mathrm{DKO}$ heart tissues when compared to the control group. The residual $5 \mathrm{hmC}$ signals could be ascribed to the existence of Tet1 or cell types other than CMs. To further delineate the function of $5 \mathrm{hmC}$ in regulating cardiac-specific gene expression, we performed CMS-IP-seq ${ }^{40,41}$ to profile genomewide $5 \mathrm{hmC}$ levels in E12.5 heart tissues (Supplementary Table 1, Supplementary Fig. 5A-B). Consistent with the IF and dot-blot results, we observed a global decrease of $5 \mathrm{hmC}$ in Tet $2 / 3-\mathrm{DKO}$ samples (Fig. 4c, Supplementary Fig. 5B). Among 9,559 identified DHMRs between the control and Tet2/3-DKO groups, we detected 8,846 genomic regions with reduced hydroxymethylation (defined as hypoDHMRs) and only 713 regions with increased DNA hydroxymethylation (designated hyperDHMRs) in Tet2/3KO heart tissues (Fig. 4c). GREAT analysis revealed that hypoDHMRs were primarily enriched at distal regulatory regions of genes, many of which are known to be important for heart development (e.g., genes involved in Notch pathways) (Fig. 4d). Next, to assess the potential biological functions correlated with DHMRs, we further analyzed histone enrichment and DNA methylation levels within the identified DHMRs using the ENCODE data from E12.5 murine heart tissues. We found that DNA methylation levels at these Tet2/3-regulated hypoDHMRs were relatively low (with median DNA methylation level at 0.30 ) (Supplementary Fig. 5C). Furthermore, these regions were highly enriched with $\mathrm{H} 3 \mathrm{~K} 4 \mathrm{mel}$ and $\mathrm{H} 3 \mathrm{~K} 27 \mathrm{Ac}$, which are usually marked at enhancers (Fig. 4e, Supplementary Fig. 5D). By contrast, the average DNA methylation of hyperDHMRs were relatively high with a median level at 0.88 (Supplementary Fig. 5C), accompanied by moderate enrichment of $\mathrm{H} 3 \mathrm{~K} 36 \mathrm{me} 3$ but no other histone marks (Fig. 4e, Supplementary Fig. 5D). Notably, DNA methylation levels at hypoDHMRs were found to undergo larger fluctuations than hyperDMRs (Supplementary Fig. 5E) during cardiac development. In summary, our unbiased epigenomic analyses suggested that Tet2/3-mediated DNA hydroxymethylation reshapes the epigenetic status of genomic regions that are important for transcriptional regulation during heart development.

Next, we aimed to address whether Tet/5hmC loss alters the DNA methylation during cardiac development. We measured DNA methylation in Tet2/3-DKO heart tissues collected at E12.5 using whole genome-wide bisulfite sequencing (WGBS) analysis ( $\sim 30 \times$ coverage of CpGs). In parallel, we compared our own Tet $2 /$ 3-KO WGBS data with ENCODE WGBS data collected with E12.5 WT heart tissues. We noted a slight increase of the average DNA methylation level ( $\mathrm{mCG}+\mathrm{hmC/CG}$ ) in the DKO group (Fig. 4f, Supplementary Fig. 5F). We next compared the DNA methylation levels at each $\mathrm{CpG}$ sites between the control and Tet2/3-DKO groups. We identified 13,377 and 27,880 hyper- or hypo-differentially methylated regions (DMRs) in the Tet2/3DKO group (Supplementary Fig. 5G). GREAT analysis showed that hyper-DMRs are enriched at genes closely associated with heart function (Fig. 4g). We further analyzed DNA methylation within identified hypoDHMRs and observed that the majority of hypoDHMRs displayed increased DNA methylation (Fig. 4h, Supplementary Fig. 5H), suggesting that Tet-mediated DNA hydroxymethylation indeed mediates DNA demethylation during cardiac development. In addition, we observed a number of hypoDMRs in Tet2/3-DKO heart tissue (Supplementary Fig. 5G) which might be due to the cross-talk between Tet proteins and Dnmt families ${ }^{42}$. Unlike hyper-DMRs, the function of these hypo-DMRs are not clear: they are not associated with genes involved in regulating cardiac function (Supplementary Fig. 5I) and are not co-enriched with histone modifications (Supplementary Fig. 5J). Further studies are needed to clarify the regulation and function of hypo-DMRs in the Tet2/3-DKO group.

In addition, we compared the alterations in DNA methylation or hydroxymethylation with changes in gene expression between WT and Tet2/3-DKO heart tissues collected from E12.5 embryos. A significant fraction of DEGs displayed increased DNA methylation $(43.1 \%)$ and decreased hydroxymethylation $(39.8 \%)$ in the Tet2/3-DKO group, respectively (Supplementary Fig. 5K). These results suggest that DNA methylation and hydroxymethylation at least partially contributed to transcriptional regulation during early heart development.

5hmC loss perturbs YY1 binding and chromatin accessibility. Tet-mediated DNA hydroxymethylation has been correlated with chromatin accessibility ${ }^{10-12}$. This prompted us to examine the genome-wide changes of chromatin accessibility by using ATACseq (Supplementary Table 1, Supplementary Fig. 6A, B) in Tet2/ 3-DKO embryonic heart. Upon Tet $2 / 3$ deletion, a total of 2816 
a

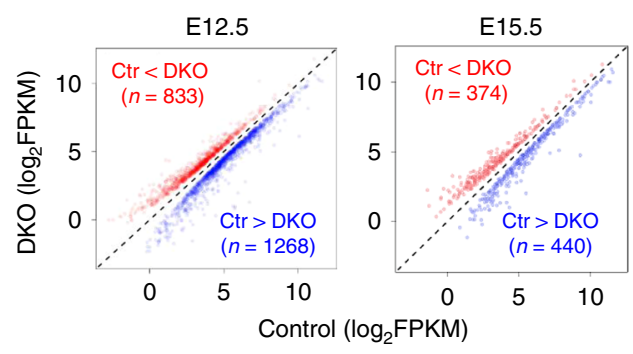

C

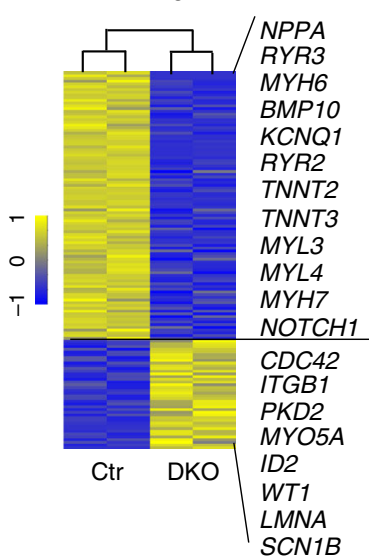

ENCODE

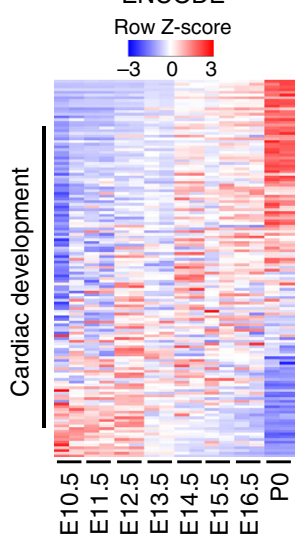

b

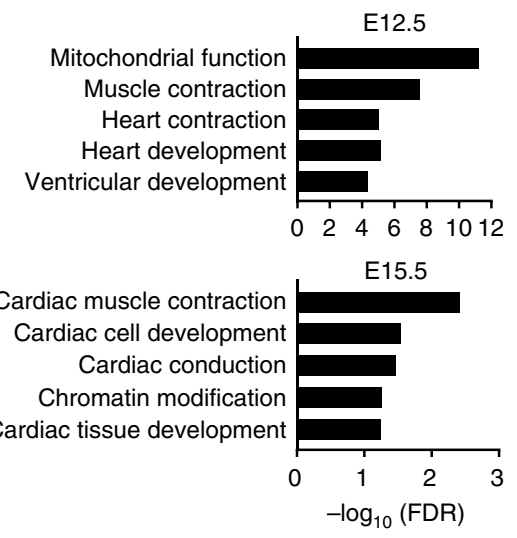

d

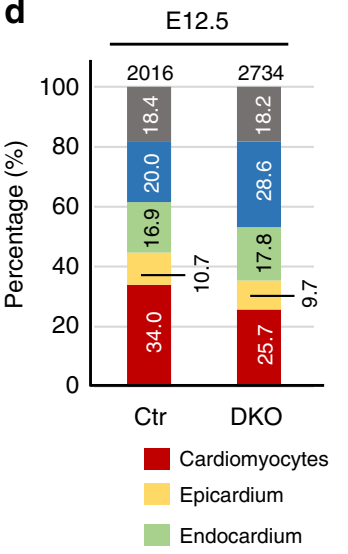

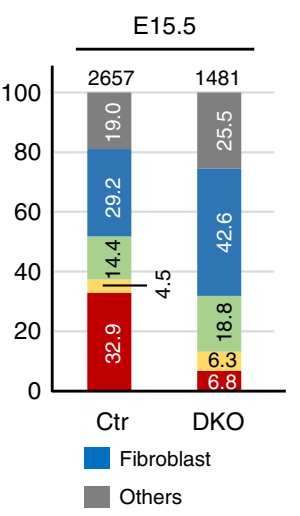

e
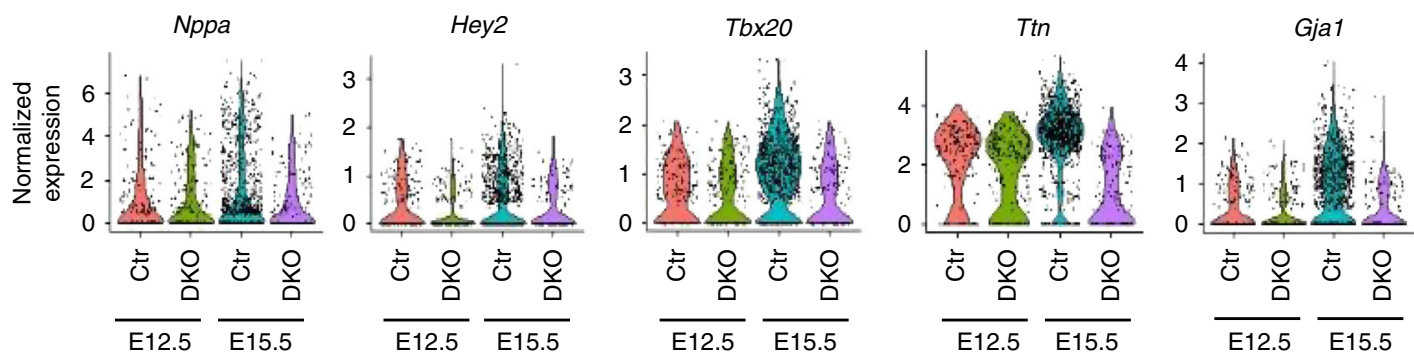

$\mathbf{f}$
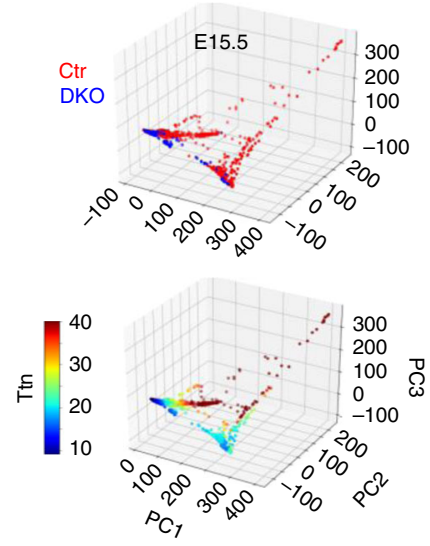

g
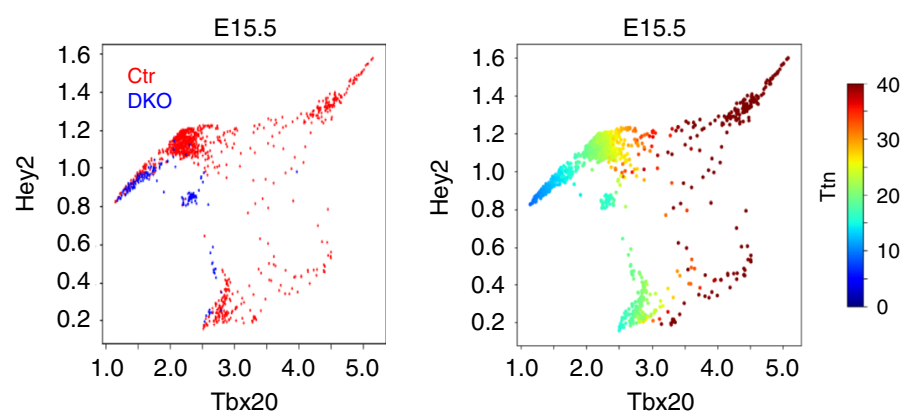

and 960 genomic regions displayed reduced and increased chromatin accessibility, respectively (Fig. 5a). Interestingly, we observed a strong positive correlation between $5 \mathrm{hmC}$ and chromatin accessible regions in both control and Tet2/3-DKO heart tissues (Pearson correlation coefficient of 0.85 ; Fig. 5b). More than $61 \%$ of ATAC-seq peaks overlapped with $5 \mathrm{hmC}$-enriched regions (Supplementary Fig. 6C). Subsequently, we selected genomic regions displaying altered $5 \mathrm{hmC}$ levels or chromatin accessibility in the Tet $2 / 3-\mathrm{DKO}$ group and found that $68.4 \%$ of selected regions showed simultaneous reduction in $5 \mathrm{hmC}$ and chromatin accessibility (Fig. 5c, Supplementary Fig. 6D). GREAT analysis revealed that these overlapping regions were mostly 
Fig. 3 Transcriptomic analyses on control and Tet2/3-DKO embryonic heart tissues. a Scatter plot of the RNA-seq expression data to identify differentially expressed genes (DEGs) in embryonic heart tissues between the control and Tet2/3-DKO groups at the E12.5 (left) or E15.5 (right) developmental stages. DEGs were defined as q-value $<=0.05$. Red and blue dots stand for up- and down-regulated genes, respectively, in the Tet2/3-DKO group when compared to control. b GSEA analysis of DEGs identified between control and Tet2/3-DKO embryonic heart tissues collected at E12.5 (top) and E15.5 (bottom). Benjamini-Hochberg corrected hypergeometric $p$-value were used. c (Left) Heatmap presentation of the cardiac development-associated DEGs in control and Tet2/3-DKO heart tissue collected at E12.5. (Right) Heatmap presentation of expression data for the same group of cardiac developmentassociated DEGs in embryonic heart tissues collected at different developmental stages (E10.5 to PO). RNA-seq data were obtained from ENCODE.

d Percentages of cell types in E12.5 and E15.5 heart tissues collected from control and Tet2/3-DKO mice using single-cell RNA-seq (scRNA-seq) analysis. Numbers listed above each bar represent the total analyzed cell numbers. e Violin plot showing the distribution of normalized expression levels of selected DEGs at E12.5 and E15.5 heart tissues collected from the control and Tet2/3-DKO groups. CMs were selected based on the expression of cTnT in each cell. Each dot represents the expression levels of corresponding genes in single cells. $\mathbf{f}$ The 3D PCA plots (top) and Ttn expression levels (bottom) of individual cardiomyocytes based on scRNA-seq data in the E15.5 control (red) and Tet2/3-DKO (blue) groups. g Selected Ttn-expressing CMs from E15.5 control (red) and Tet2/3-DKO (blue) were displayed based on the expression levels of Hey2 and Tbx20 (left). The expression of Ttn in the corresponding cells were shown in the right panel

enriched at distal regulatory regions of genes important for heart development (e.g., Mly2, Tnnt2, and Ttn; Supplementary Fig. 6E). Motif analysis further identified the enrichment of transcription factors (TFs) binding motifs for key cardiac development genes, such as Mef2C, Gata4 and Nkx2.5 (Supplementary Fig. 6F), within these genomic regions. We next examined the effects of decreased 5hmC and ATAC on transcription level and observed positive correlation between gene expression and 5hmC/ATACseq signals. For instance, we observed a strong association between reduced expression of cardiac development genes (e.g., Nppa, Tnni2, and Bmp10) and reduced 5hmC/ATAC-seq signals within $2 \mathrm{~kb}$ of transcription start sites (TSS) at corresponding genes (Fig. 5d, e, Supplementary Fig. 6G). Taken together, these data suggest that cardiac specific deletion of Tet proteins induced $5 \mathrm{hmC}$ loss and caused a reduction in chromatin accessibility to alter cardiac gene expression.

Based on the data described above, we hypothesized that Tet deletion reduces $5 \mathrm{hmC}$ and chromatin accessibility, and subsequently affects key TF binding to their genomic targets in embryonic hearts. GREAT analysis on ATAC-seq data from control and Tet2/3-DKO heart tissues pointed to YY1 as the top candidate, which showed strong enrichment in genomic regions displaying reduced chromatin accessibility in the Tet $2 / 3-\mathrm{DKO}$ group (Fig. 5f). YY1, a member of the Gli-Kruppel family of zinc finger protein, is an important transcription factor regulating early heart development ${ }^{43}$. To test this hypothesis, we generated Tet triple knockout mouse embryonic stem cells (Tet-TKO mESC) by using the CRISPR/Cas9-based genome editing tool as previously reported ${ }^{44}$ (Supplementary Fig. 7A-C). Since TetTKO mESC has an undetectable 5 hmC level (Supplementary Fig. 7B), it provides a clean system to elucidate the impact of $5 \mathrm{hmC}$ on TF binding to chromatin. Next, we measured the chromatin association of YY1 in WT and Tet-TKO mESCs. We observed a significant decrease in chromatin associated YY1 in Tet-TKO mESCs compared with parental WT mESCs (Fig. 6a, Supplementary Fig. 8A).

To further examine the correlation between $5 \mathrm{hmC}$ and YY1, we applied the CUT\&RUN (C\&R) $\operatorname{method}^{45}$ to enrich YY1 genomic binding regions in WT and Tet-TKO mESCs. We first compared YY1 enriched regions obtained from the C\&R method with published YY1 ChIP-seq data in mESCs $^{46}$ and observed a similar pattern between these two datasets (Supplementary Table 1, Supplementary Fig. 8B, C), revealing the robustness of the YY1 C\&R data. Then we examined the correlation between YY1 and $5 \mathrm{hmC}$ in parental WT mESCs. We observed a strong $5 \mathrm{hmC}$ enrichment with concomitant depletion of $5 \mathrm{mC}$ signals at YY1 enriched regions (Fig. 6b). About 16\% of YY1-enriched peaks $(n=10,450)$ overlapped with $5 \mathrm{hmC}$ enriched regions (Fig. 6c). To further examine the impact of Tet $/ 5 \mathrm{hmC}$ loss on
YY1 genomic distribution, we compared YY1 enriched regions between the WT and Tet-TKO groups and noted that $73 \%$ of YY1 peaks showed less enrichment in Tet-TKO mESCs (Fig. 6d, e, Supplementary Fig. 8D-E), particularly at genomic regions that displayed $5 \mathrm{hmC}$ reduction upon Tet deletion (Supplementary Fig. 8F). We also observed increased DNA methylation within YY1 binding regions in Tet-TKO mESCs (Fig. 6f). To further validate this in a physiologically-relevant context, we performed YY1 C\&R in heart tissues collected from E12.5 control and Tet2/ 3 -DKO mice. Due to the limited cell numbers in the tissue, we identified slightly lower numbers $(n=11,469)$ of high-confident YY1-enriched regions when compared with the YY1 peak numbers in mESCs $(n=15,055)$. Using these high confident YY1 enriched peaks, we found a strong reduction of YY1 enrichment in Tet2/3-DKO heart tissues (Fig. 6g, Supplementary Fig. 8G), which is consistent with results made from the mESC study (Fig. 6d, e). Likewise, we observed increased DNA methylation and reduced $5 \mathrm{hmC}$ enrichment within YY1enriched regions in the Tet2/3-DKO group (Supplementary Fig. $8 \mathrm{H}, \mathrm{I})$. To further examine the impact of chromatin accessibility on YY1 binding, we compared YY1 enriched regions with ATAC-seq signals in both the control and Tet2/3-DKO groups. We noted a strong reduction of YY1 enrichment at genomic regions with reduced chromatin accessibility measured by ATAC-seq (Fig. 6h). These data further confirmed that Tet and $5 \mathrm{hmC}$ regulate chromatin accessibility to facilitate the binding of proper TFs, such as YY1, to their targets.

5hmC loss disrupts higher-order chromatin structures. YY1 is known to regulate chromatin higher-order structures by controlling promoter-enhancer looping ${ }^{47}$. In addition, together with others, we have reported that $5 \mathrm{hmC}$ is enriched at euchromatin $^{11-13}$. Chromatin is known to be spatially categorized into two types of large compartments, $\mathrm{A}$ and $\mathrm{B}$, that exhibit either open chromatin domain (A) or closed chromatin domain $(B)^{48}$. To examine whether Tet mediated DNA hydroxymethylation is associated with the organization of chromatin higher-order structures, we performed HiChIP experiment in control and Tet2/3-DKO E12.5 heart tissues using an anti-smc1 antibody ${ }^{49}$ (Supplementary Table 1, Supplementary Fig. 9A, B). Interestingly, we observed a strong enrichment of $5 \mathrm{hmC}$ in compartment $\mathrm{A}$, but not in compartment B (Fig. 7a, Supplementary Fig. 9C), suggesting that $5 \mathrm{hmC}$ tends to mark transcription active regions. Then we compared three-dimensional chromatin interaction patterns between control and Tet2/3-DKO heart tissues. In general, the compartment organization between control and Tet $2 / 3$ DKO hearts showed very similar patterns $(R=0.93$; Fig. $7 b)$. However, we observed 1424 bins (50 kb resolution) switching 
a

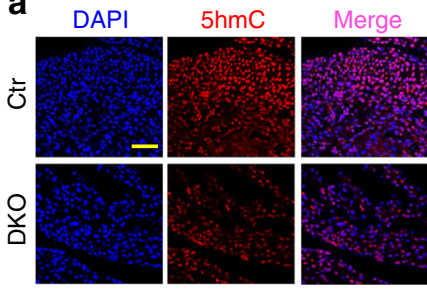

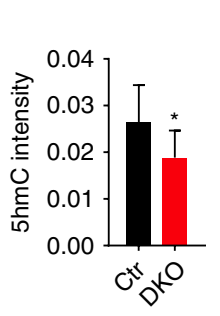

b

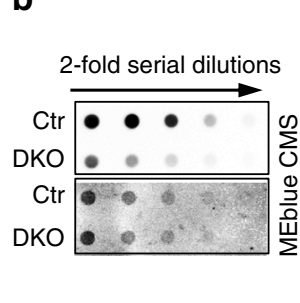

C

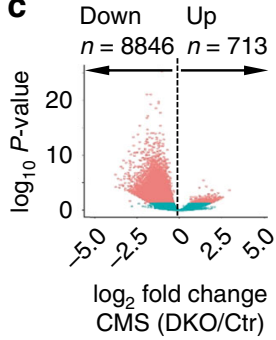

d

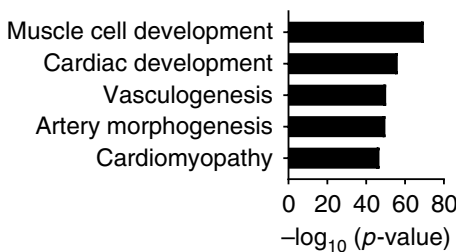

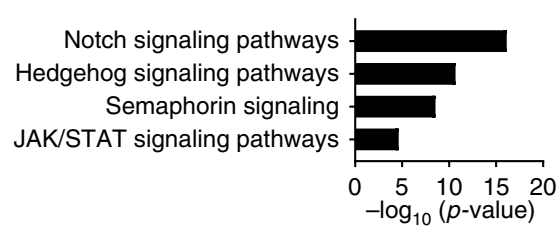

e

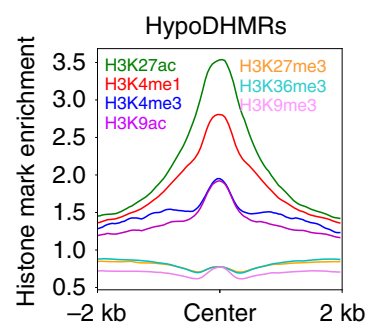

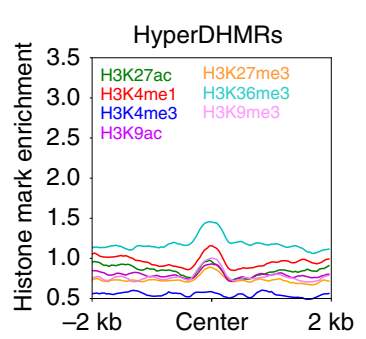

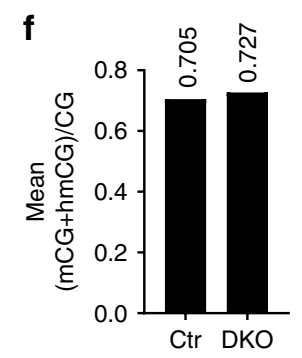

g

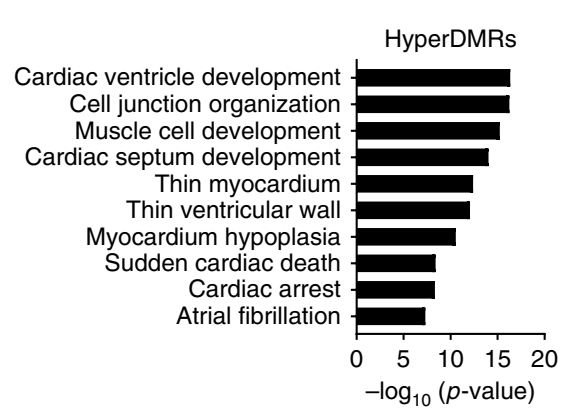

$\mathbf{h}$

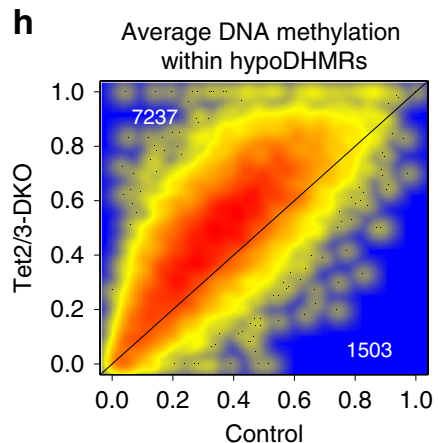

Fig. 4 Tet2/3 deletion in embryonic heart resulted impaired $5 \mathrm{hmC}$ but not $5 \mathrm{mC}$. a (Left) Representative IF staining images for control and Tet2/3-DKO murine heart tissues collected at E12.5. Blue, DAPI; Red, 5hmC. (Right) Quantification of 5hmC levels in control and Tet2/3-DKO heart tissues collected at E12.5. Data were shown as mean \pm S.D; $n=3$ independent experiments (a total of 1038 and 1250 cells were analyzed for control and Tet2/3-DKO, respectively). ${ }^{\star \star} p<0.01$ compared to control (two-tailed Student's t-test were used). Scale bar: $50 \mu \mathrm{m}$. b Measurement of the global $5 \mathrm{hmC}$ levels in control and Tet2/3-DKO heart tissues collected at E12.5 by using the dot-blot assay. Methylene blue (MEblue; bottom) staining was used to visualize the total DNA input. c Volcano plot illustrating the differentially enriched $5 \mathrm{hmC}$ regions (DHMRs) in E12.5 heart tissues between the control and Tet2/3-DKO groups ( $p$ value $<=0.05$ ). The mean size of hypoDHMRs was $593 \mathrm{bp}$, covering $0.18 \%$ of the genome. $\mathbf{d}$ Representative GREAT analysis on hypoDHMRs illustrated in Fig. 4c. Corrected binomial raw p-value were calculated. e Normalized enrichment of the indicated histone modifications within identified hypoDHMRs (left) and hyperDHMRs (right). f Average DNA methylation levels in control and Tet2/3-DKO murine heart tissues collected at E12.5. g GREAT analyses on identified top 5000 most significant changed hyperDMRs in Tet2/3-DKO heart tissues compared with control. Corrected binomial raw $p$-value were calculated. $\mathbf{h}$ Scatterplot of the average DNA methylation levels within hypoDHMRs in E12.5 control and Tet2/3-DKO heart tissues

from compartment A to B upon Tet $2 / 3$ depletion in heart tissues (Fig. 7b). Next, we evaluated the expression levels of genes that fell into the A-to-B compartment switch category. We identified 250 down-regulated genes, with many of them known to be important for heart development, such as Ttn, Cav1, Bmp5, and Actc1 (Fig. 7c); Go Ontology (GO) analysis showed that these genes are important for maintaining normal heart function or are closely implicated in cardiomyopathies (Fig. 7d).

Next, we calculated unique paired-ended tags (PETs) using the Fit-HiC pipeline ${ }^{50}$ to identify the long-distance contacts in E12.5 heart tissues. We identified 475,630 and 347,816 confident contacts in control and Tet $2 / 3-\mathrm{DKO}$ heart tissues, respectively (Fig. 7e). We also observed strong positive association among PETs, 5hmC enriched regions and chromatin accessible regions (Supplementary Fig. 9D). We further moved on to examine the potential impact of altered contacts on gene transcription by counting PETs at DEGs identified between the control and Tet2/ 3 -DKO groups. We noticed that $60 \%(n=979)$ of DEGs with mapped PETs exhibited reduced long distance contacts in the Tet2/3-DKO group (Fig. 7f). For example, at the Tbx20 and Hey2 loci, two genes which displayed significant downregulation upon Tet deletion in scRNA-seq analysis, we detected a pronounced 


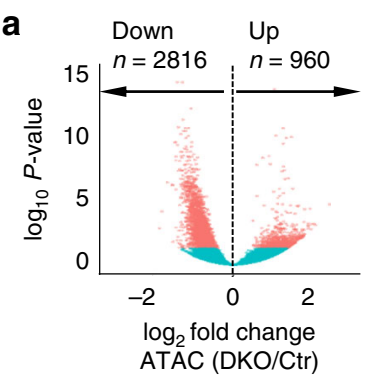

C

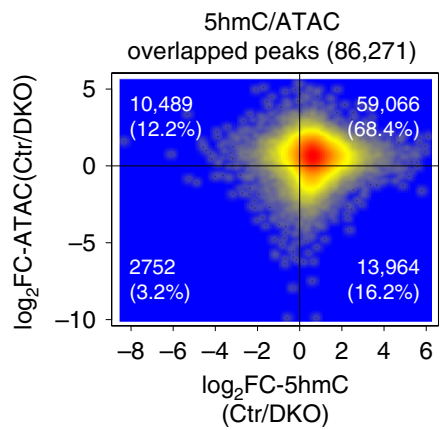

b

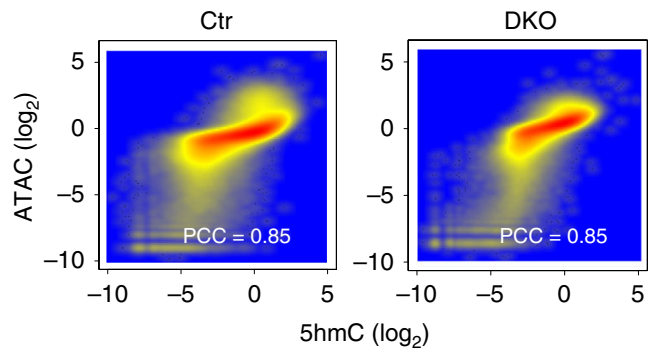

d

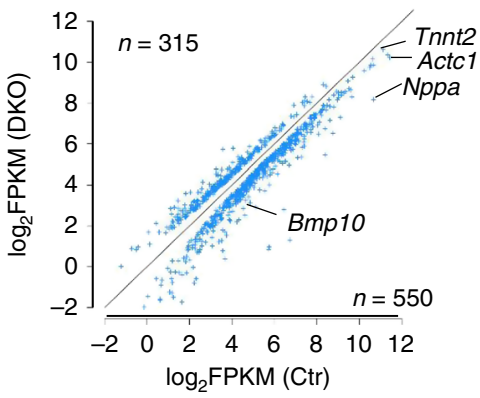

f e

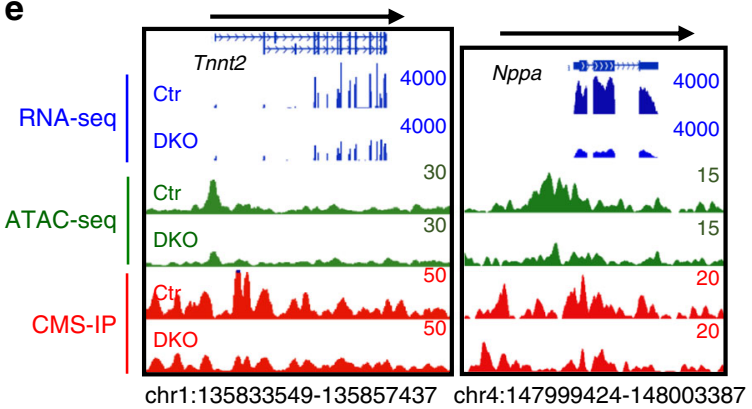

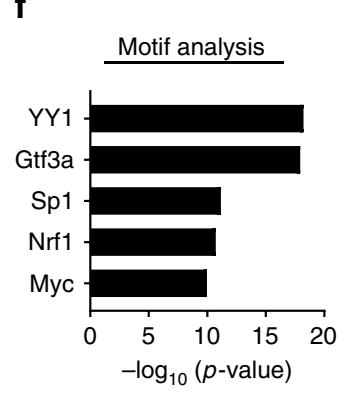

Fig. 5 Tet2/3 deletion in murine embryonic hearts reduced chromatin accessibility. a Volcano plot depicting the differential ATAC-seq enriched regions between the control and Tet2/3-DKO groups ( $p$ value $<=0.05$ ). Data were collected from E12.5 embryonic heart tissues obtained from control and Tet2/ 3-DKO mice. b Scatterplot depicting the correlation between $5 \mathrm{hmC}$ and ATAC-seq signals in the control (left) and Tet2/3-DKO (right) groups. Pearson correlation coefficient was calculated by correlating $5 \mathrm{hmC}$ signals with ATAC-seq signals obtained from E12.5 cardiac tissues. The ATAC and $5 \mathrm{hmC}$ signals of every $10 \mathrm{~kb}$ bin were calculated. c Scatterplot depicting the distribution of genomic regions with altered $5 \mathrm{hmC}$ ( $x$-axis) and ATAC-Seq signals ( $y$-axis) between the control and Tet2/3-DKO groups. Normalized 5hmC and ATAC signals were used in the plot. d Scatterplot presenting the expression of DEGs that showed decreased in $5 \mathrm{hmC}$ and ATAC-seq signals within $2 \mathrm{~kb}$ of transcription start sites (TSS). Tnnt2, Actc1, Nppa, and Bmp10 were among the hits. e Genome browser view examples of decreased transcription (blue, RNA-seq), chromatin accessibility (green, ATAC-seq) and 5hmC (red, CMS-IP) enrichment at cardiac-specific gene loci (Tnnt2 and Nppa) in Tet2/3-DKO E12.5 heart tissues compared with that in control group. $\mathbf{f}$ Motif enrichment analysis on genomic regions displaying decreased ATAC-seq signals in the Tet2/3-DKO group. Benjamini-Hochberg corrected hypergeometric $p$-value were used

decrease of promoter-enhancer interactions in the Tet2/3-DKO group compared to the control group (Fig. $7 \mathrm{f}, \mathrm{g}$ ). In parallel, we noted a concomitant reduction in $5 \mathrm{hmC}$ and ATAC-seq signal enrichment in the Tet2/3-DKO group within these two loci (Fig. 7g, Supplementary Fig. 9E). To further evaluate the impact of Tet $/ 5 \mathrm{hmC}$ in regulating YY1 binding during the long-distance interaction, we compared Hi-ChIP signals between WT and TetTKO mESCs (Supplementary Fig. 9F, G). We observed a strong reduction of PETs at YY1 enriched regions in Tet-TKO mESCs compared to the parental WT ESCs (Supplementary Fig. 9G). In parallel, we carried out a functional rescue experiment by expressing the catalytic domain of Tetl (Tet1CD) in WT or Tet-TKO mESCs. The expression of Tet1CD and subsequent increase of $5 \mathrm{hmC}$ in the Tet-TKO mESCs were confirmed by Western blotting (Supplementary Fig. 9H) and the $5 \mathrm{hmC}$ dotblot assay (Supplementary Fig. 9I). Next, YY1 ChIP-qPCR and $4 \mathrm{C}$-seq were performed at selected genomic regions to examine
YY1 binding and the chromatin looping status before and after Tet1CD re-expression (Fig. 8a, b). We selected the Mpdu1 locus because it was among the top ranked genomic regions with decreased YY1 binding and chromatin looping upon Tet depletion. We found that Tet1CD expression in Tet-TKO mESCs significantly restored YY1 binding (Fig. 8a) and chromatin looping (Fig. 8b) at the Mpdu1 locus, suggesting the involvement of Tet $/ 5 \mathrm{hmC}$ in regulating YY1-associated long-range chromatin interactions in the genome. Together, these findings establish that Tet proteins modulate the formation of YY1-associated promoteenhancer looping and use this mechanism to regulate gene transcription.

\section{Discussion}

Abnormal DNA methylation has been reported in multiple congenital heart diseases, suggesting that dynamic DNA 
a
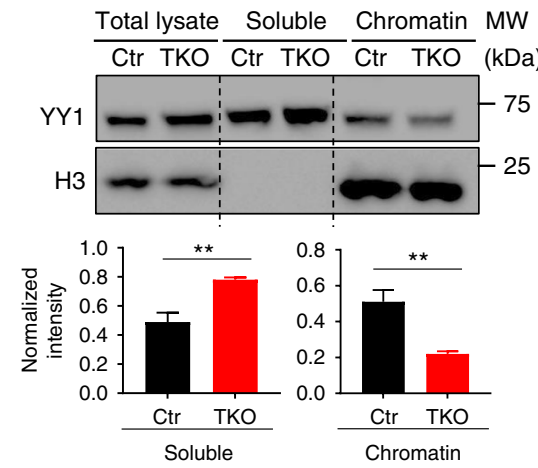

C

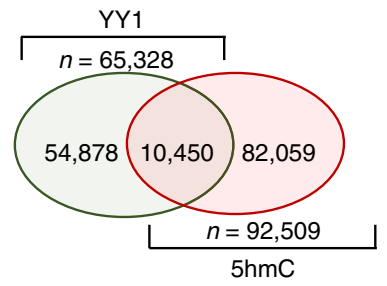

e
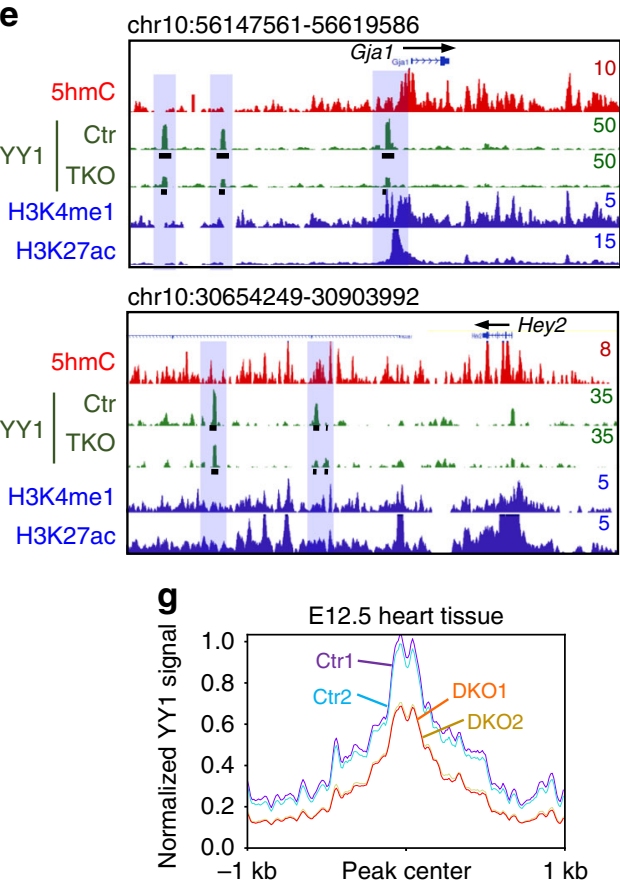
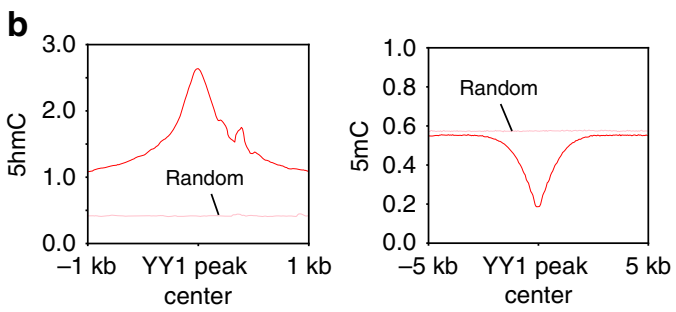

d
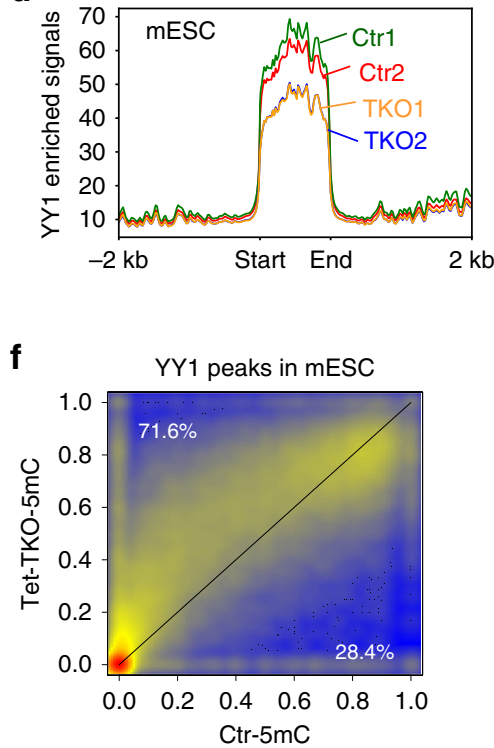

h

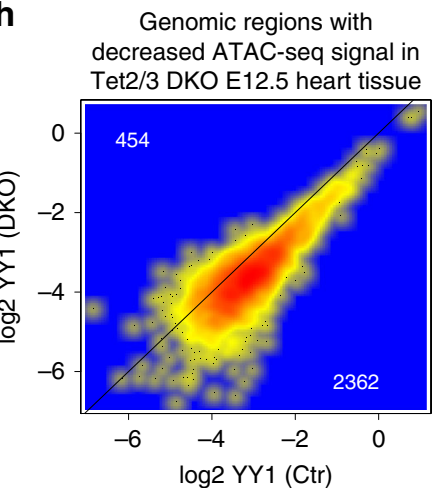

Fig. 6 Tet2/3 deletion compromised the binding of $Y Y 1$ to chromatin. a (Top) Representative western-blot analysis of $Y Y 1$ binding to chromatin in WT and Tet-TKO mESCs. (Bottom) Quantification of the intensity of YY1 soluble and chromatin associated fractions in WT and Tet-TKO mESCs. Data were shown as mean \pm S.D; $n=3$ independent experiments. ${ }^{\star \star} p<0.01$ compared to WT (two-tailed Student's $t$-test were used). $\mathbf{b}$ Enrichment of $5 \mathrm{hmC}$ (left) and $5 \mathrm{mC}$ (right) signals within YY1-enriched peaks. Random regions were used as control. c Venn diagram showing overlapped peaks between YY1- and 5hmCenriched regions in mESCs. 10,450 overlapping peaks were identified. d YY1 enrichment in WT (red and green) and Tet-TKO (yellow and blue) mESCs. e Exemplary genome-browser views of $5 \mathrm{hmC}$ enrichment in WT mESCs (red trace) and YY1 enrichment (green) at the Gja1 and Hey2 loci in WT and TetTKO mESCs. Representative genomic regions that showed reduction in YY1 enrichment in Tet-TKO mESCs (compared to control) were highlighted in blue. The black bars beneath the peaks indicated the called peaks. $\mathbf{f}$ Scatterplot depicting the DNA methylation levels within the YY1 enriched regions in WT ( $x$-axis) and Tet-TKO ( $y$-axis) mESCs. $\mathbf{g}$ YY1 enrichment in control (blue and purple) and Tet-DKO (yellow and orange) mouse embryonic heart tissues collected at the E12.5 stage. $\mathbf{h}$ Scatterplot depicting YY1 enrichment within the genomic regions that showed decreased ATAC-seq signals in control $(x$-axis) and Tet-DKO ( $y$-axis) heart tissues collected at E12.5 mouse embryos

methylation is one of the important epigenetic events controlling heart development and cardiac functions ${ }^{19-21}$. In the current study, we have systematically analyzed DNA methylation and hydroxymethylation dynamics during embryonic heart development in both human and rodents. Our integrative studies have unveiled dynamic focal DNA methylation changes, driven by
TET-mediated DNA hydroxymethylation, at genes essential for cardiac development.

The TET protein family is one of the major regulators controlling DNA methylation oxidation. The current study is primarily focused on studying Tet2 and Tet3 given their relatively higher expression in embryonic cardiac tissues. Cardiac specific 
a

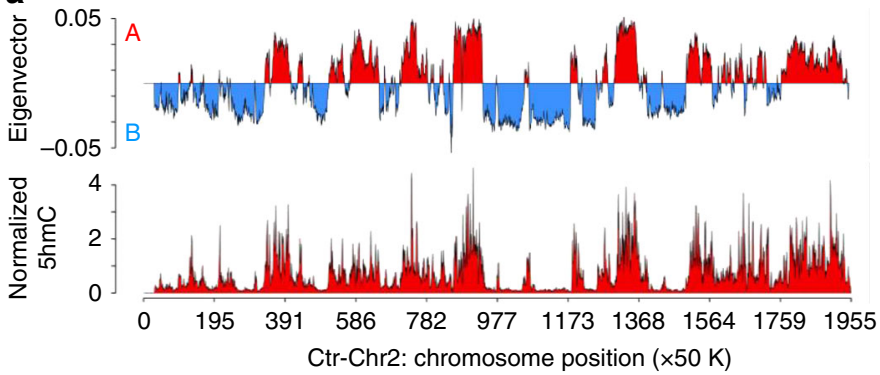

b

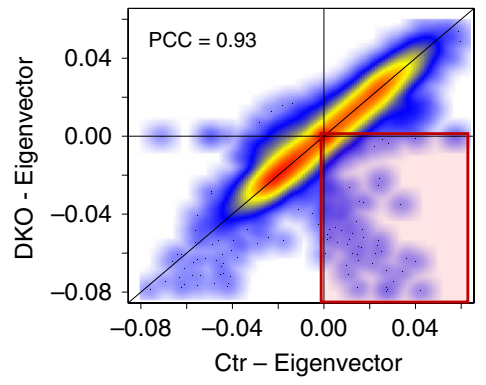

c

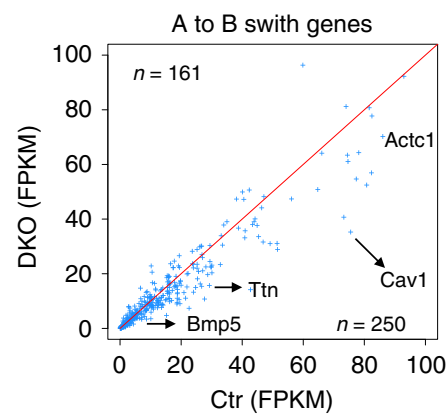

e

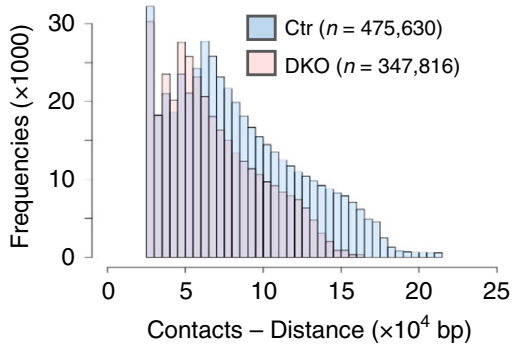

f

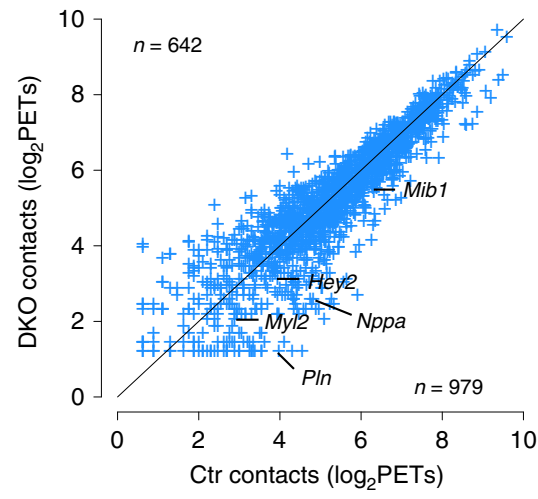

d

Hypertrophic cardiomyopathy Arrhythmogenic cardiomyopathy

Dilated cardiomyopathy Adherens junction Cardiac muscle contraction

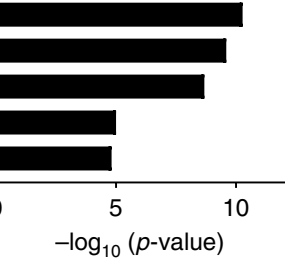

g

chr9:24555304-25037297
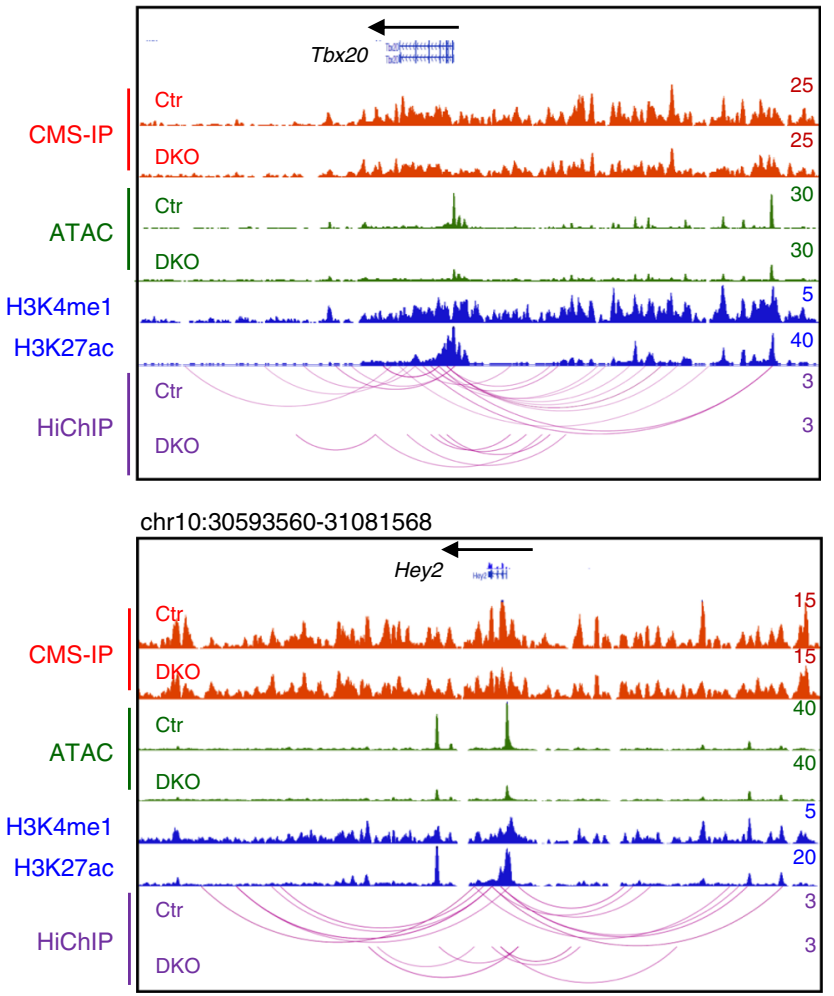

Fig. 7 Tet-mediated DNA hydroxymethylation is associated with chromatin higher-order structures. a Representative histograms of compartment A and B distribution (top) calculated by Eigenvector using HiChIP and CMS-IP-seq (for 5hmC) data over chromosome 2 in E12.5 control embryonic heart tissues. b Scatterplot showing the distribution of Eigenvector calculated in control and Tet2/3-DKO embryonic heart tissues at E12.5. Pearson correlation coefficient was calculated. c Expression levels of genes that underwent A-to-B compartment switch in control and Tet2/3-DKO embryonic heart tissues (E12.5). d Representative GSEA analysis of genes located in genomic regions that showed A-to-B compartment switch in Tet2/3-DKO embryonic heart tissues (E12.5). e Histograms of PETs (distance and numbers) from HiChIP data obtained from control and Tet2/3-DKO heart tissues collected at E12.5. f Scatterplot showing the normalized numbers of PETs that overlapped with DEGs (regions ranging from TSS to TTS) in control and Tet2/3-DKO heart tissues collected at E12.5. $\mathbf{g}$ WashU Epigenome browser views of 5hmC, ATAC-seq, PETs (HiChIP), H3K4me1 (ENCODE) and H3K27ac (ENCODE) at the Tbx2O and Hey2 loci in control and Tet2/3-DKO heart tissues collected at E12.5 
a

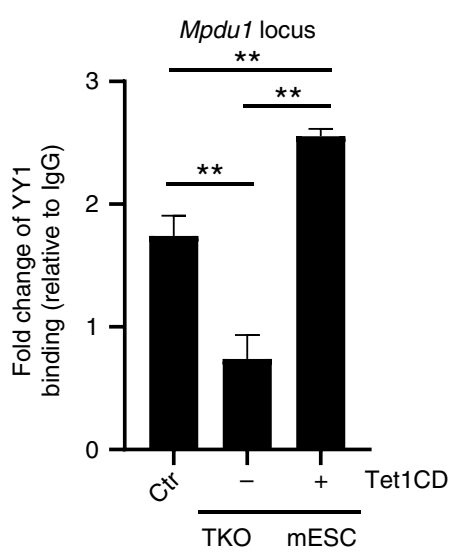

b

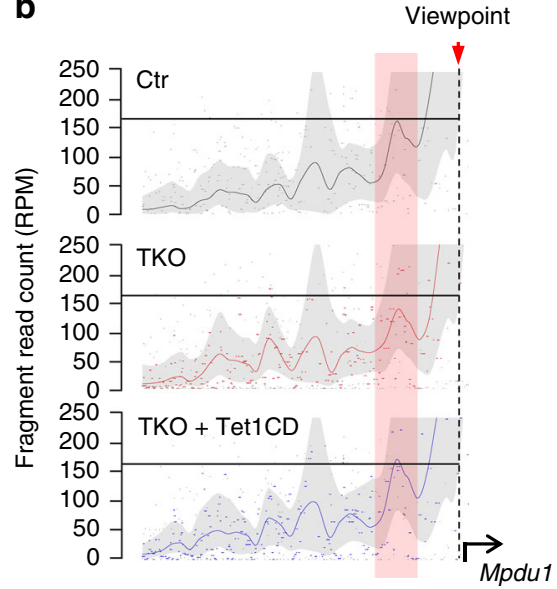

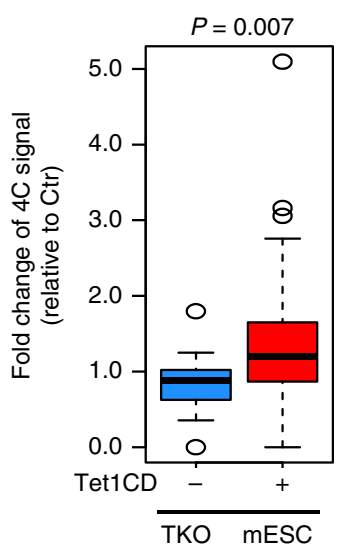

C

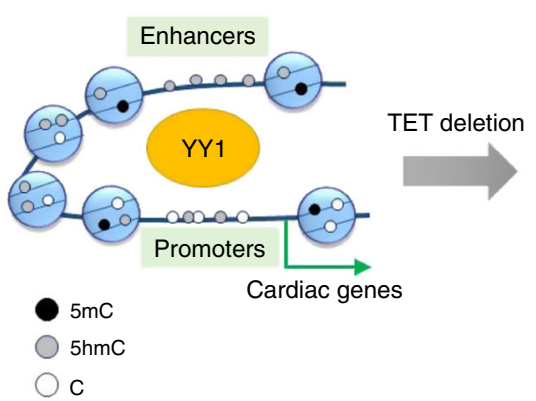

$\downarrow 5 \mathrm{hmC}$

$\downarrow$ Chromatin accessibility
Disrupt enhancerpromoter looping
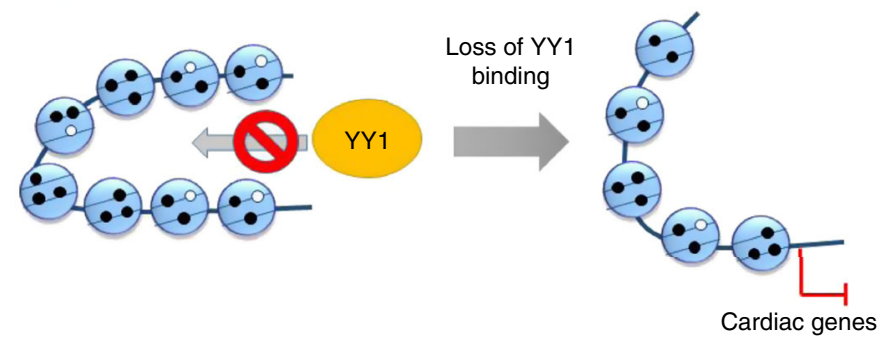

Fig. 8 Re-expressing Tet1CD partially restores the YY1 mediated enhancer-promoter interactions. a ChIP-qPCR analysis of YY1 binding at the Mpdu1 locus in TKO mESCs and TKO mESCs after expression of Tet1CD. Data were shown as mean \pm S.D; $n=2$ independent experiments. ${ }^{\star \star} p<0.005$ (two-tailed Student's $t$-test were used). b Representative 4C-seq signals in WT (black), TKO (red) and TKO + Tet1CD (blue) mESCs at the Mpdu1 locus. The line indicates the normalized 4C-seq signals (calculated by Basic4C-seq R package using two biological replicates) and the shaded areas represent the $95 \%$ confidence interval. The quantification of $4 \mathrm{C}$ signals at red highlighted regions were shown in the right panel. Blue: relative fold-change of $4 \mathrm{C}$ enrichment between TKO and WT (TKO/WT); Red: relative fold-change of $4 \mathrm{C}$ enrichment between TKO + Tet1CD and WT (TKO + Tet1CD/WT). All the experiments were performed with biological duplicates. Kolmogorov-Smirnov test were used to calculate $p$-value. c Tet protein mediated DNA hydroxymethylation regulates chromatin accessibility and subsequently safeguards the binding of key cardiac development-associated transcription factors, such as $Y Y 1$, to their target regions in the genome to maintain proper long distance interactions (enhancer-promoter looping). Deletion of Tet protein, with consequent $5 \mathrm{hmC}$ loss, could reduce chromatin accessibility to compromise $\mathrm{YY} 1$ binding to its genomic targets, thereby affecting long distance interactions to perturb the transcriptional networks underlying normal cardiac development

deletion of Tet2 and Tet3 using Nkx2.5-Cre resulted in ventricular non-compaction cardiomyopathy in mice, clearly attesting to the indispensable roles of these two epigenetic modifiers in normal heart development. At the molecular level, deletion of Tet $2 / 3$ prominently impaired DNA methylation/hydroxymethylation in heart tissues and altered the transcription of cardiac development associated pathways, such as Notch signaling. Mechanistically, we propose that changes in chromatin accessibility, attributed to compromised DNA hydroxymethylation, sabotage the binding of key cardiac development-associated TFs, as exemplified by YY1 (Fig. 8c), to their targets across the genome. YY1 is a key mediator of embryonic heart development by facilitating GATA4 mediated transcriptional activation and promoting cardiac progenitor cell commitment ${ }^{43,51}$. Interestingly, cardiac-specific $\mathrm{YY} 1$ knockout mice $\left(\mathrm{YY}^{\mathrm{f} / \mathrm{f}}-\mathrm{Nkx} 2.5 \mathrm{Cre}\right)$ displayed very similar phenotypes as Tet2/3-DKO mice generated in the current study, with both in vivo models showing reduced embryo survival at E13.5 and decreased proliferation of CMs (with similar non-compaction cardiomyopathy manifestations) ${ }^{52}$. These findings strongly suggest that Tet proteins and YY1 might converge to regulate the similar transcription regulatory pathways during embryonic cardiac development. Although we presented evidence to support the notion that Tet deletion results in reduced chromatin binding of YY1 because of altered chromatin accessibility, we cannot rule out the possibility that the binding of YY1 to the genome is directly dependent on Tet and /or DNA hydroxymethylation modifications. Further follow-on studies are needed to clarify this point.

Although we observed a positive correlation between YY1 binding and Tet-mediated DNA hydroxymethylation, a substantial number of $5 \mathrm{hmC}$ - or YY1-enriched regions do not overlap with each other, indicating that $5 \mathrm{hmC}$ might not be the only factor to regulate YY1 genomic binding. YY1 has been reported to interact with several other epigenetic regulators, such as HDACs, p300 and INO80, to enhance or repress gene transcription ${ }^{53}$. In parallel, Tet and $5 \mathrm{hmC}$ might further be implicated in other transcriptional regulatory pathways during embryonic development. For example, Tet-mediated DNA demethylation has been reported to control the lefty-nodal signaling during mouse gastrulation ${ }^{2}$ and enhancer activities in the vertebrate phylotypic period ${ }^{11}$. Moreover, Tet1 and Tet3 deficiency has been shown to promote transcription variations during embryogenesis ${ }^{1}$. Tet 1 further regulates the activity of JMJD8 to suppress epiblast target genes in postimplantation mouse embryos ${ }^{54}$. A complete picture of the context-dependent YY1/TET/5hmC genomic distribution and 
transcriptional regulation during early embryogenesis is yet to be fully established.

In summary, our integrative genomic and epigenomic analyses have yielded a complete atlas of DNA methylomes and hydroxymethylomes representative of key developmental stages of embryonic hearts in both humans and rodents. The epigenetic landscapes depicted in our study can serve as a useful blueprint and starting point towards the full comprehension of cardiac epigenetics during early embryogenesis. Our study has uncovered previously unrecognized roles of Tet and $5 \mathrm{hmC}$ in gene regulation by modulating transcription factor binding and longdistance interactions at cardiac-specific genomic loci. In addition to Tet2 and Tet3, we also observed dynamic changes of Tet1 expression during heart development in both human and rodents. We cannot rule out the possibility that Tet1 is also an important contributor to embryonic heart development. Nonetheless, no heart-related phenotypes have thus far been reported upon Tet1 deletion in transgenic mice, probably due to the redundant functions of Tet homologs (Tet2 and Tet3). Further studies on a cardiac-specific Tet triple knockout mouse model might address the additional function of Tet1 during embryonic heart development. Although the current study exclusively focused on dissecting cell-autonomous mechanisms on how Tet protein might regulate the transcription of key genes involved in early cardiomyocytes development, we cannot rule out the possibility of cell non-autonomous mechanisms (such as how Tet deficiency in epicardium affect cardiomyocytes development) underlying this phenotype, which will be pursued in the follow-on studies.

\section{Methods}

Animal models. Animal studies were approved by the Institutional Animal Care Use Committee (IACUC) of the Institute of Biosciences and Technology, Texas A\&M University. Most mouse strains bear a C57BL/6 genetic background unless otherwise noted. Tet $2^{[-/-33}$, Tet $3 \mathrm{f} / \mathrm{f}^{1}$ and Nkx2.5-Cre (The Jackson Laboratory $024637)^{55}$ mouse strains were reported previously. Timed pregnancies were applied and the day on which a plug was found was defined as E0.5. Mice tails were cut and boiled in $50 \mathrm{mM} \mathrm{NaOH}$ for $1 \mathrm{~h}$ and then neutralized in $10 \mathrm{mM}$ Tris-HCL at pH7.4. PCR was carried out using the EmeraldAmp GT PCR Master Mix (TaKaRa) according to the manual. Genotyping primers are listed in Supplementary Table 2. The uncropped and unprocessed scans of the blots are available in source data file.

Antibodies. For IHC: Tet1 antibody was kindly provided by Dr. Leonhardt Heinrich $^{56}$ (1:100). Anti-Tet2 (Abcam ab124297, 1:100), anti-5mC (Millipore MABE146, 1:1000), anti-5hmC (Active Motif 39769, 1: 40,000), anti-5fC (Active Motif 61223, 1:2000), and anti-5caC (Active Motif 61225, 1:1000) were purchased from commercial sources.

For IF: anti-Ki67 (Abcam ab16667, 1:100), anti-cleaved caspase-3 (Cell Signaling Technologies $9661 \mathrm{~s}, 1: 50$ ); Alexa Fluor 568 goat anti-rabbit (Thermo Fisher Scientific A-11011, 1:1000), Alexa Fluor 647 goat anti-mouse (Thermo Fisher Scientific A-21235, 1:1,000)

For CUT\&RUN experiments: anti-YY1 (Santa Cruz sc-7341, 1:100), antiH3K27ac (Abcam ab4729, 1:100), Rabbit anti-mouse (Abcam ab6728, 1:100), anticTNT (Thermal Fisher Scientific 13-11, 1:100) were purchased.

For HiChIP: anti-Smc1 (A300-055A, Bethyl Laboratories, Inc. $2 \mu \mathrm{g} / \mathrm{reaction})$ was used.

For Western Blotting: anti-YY1 (Santa Cruz sc-7341, 1:1000), and anti-H3 (abcam ab1791, 1:2000) antibodies were used.

Human Samples. Human embryos collecting protocol was approved by the Ethical Internal Review Board of the Xinhua Hospital, Shanghai, China. Human embryos were collected from pregnant mothers who performed clinical drug abortion at the Department of Obstetrics and Gynecology in Xinhua Hospital. Consent forms were signed. The embryonic stages of the embryos were measured by using a standard protocol reported previously ${ }^{57}$.

Histological analyses. All mouse embryos were dissected in phosphates buffered saline (PBS). Embryos pictures were taken using a Nikon SMZ800N dissecting microscopy. For histological characterization, all embryos were fixed overnight in $4 \%$ PFA, then dehydrated with graded ethanol and embedded in paraffin. Sections were cut at the thickness of $7 \mu \mathrm{m}$. Slides were dried at $37^{\circ} \mathrm{C}$ overnight and then stained with hematoxylin-eosin (H\&E), as previously described ${ }^{58,59}$. Stained sections were imaged using a Nikon Eclipse Ci microscopy.
ImageJ was used for the measurement and quantification of histological data. The quantification method was described previously ${ }^{38}$. Briefly, the ventricle was divided into the apex and the basal regions and several measurements of the compact myocardium thickness were taken for each region. The average thickness was calculated. The trabecular area was measured by dividing the surface occupied by the trabeculae in the ventricle. The ratio of the trabecular area and compact myocardium thickness was used as an indicator for the size of the trabecular mesh.

IHC and IF staining. Immunohistochemistry (IHC) and immunofluorescence (IF) staining were performed, as previously described ${ }^{58,59}$. Briefly, tissue sections were dewaxed in xylene twice for $5 \mathrm{~min}$ each and rehydrated in a graded series of ethanol (100 to $70 \%$ ). The antigens were retrieved by boiling sections in $10 \mathrm{mM}$ citrate buffer (Vector Laboratories) for $20 \mathrm{~min}$. For DNA modifications staining, sections were treated with $2 \mathrm{~N} \mathrm{HCl}$ for $30 \mathrm{~min}$ to expose the epitopes and then neutralized in $100 \mathrm{mM}$ Tris- $\mathrm{HCl}$ ( $\mathrm{pH}$ 8.5) for $10 \mathrm{~min}$. IHC was using the ImmPRES HRP Reagent Kit (Vector Laboratories) to perform the blocking and antibody incubation, and then developed by using the $\mathrm{DAB}$ peroxidase substrate kit (Vector Laboratories). IHC stained sections were imaged by a Nikon Eclipse Ci microscopy. For IF, $10 \%$ normal goat serum (Thermo Fisher Scientific) was used to block the unspecific antigens. After primary and $2^{\text {nd }}$ antibodies incubation, $0.5 \mu \mathrm{g} / \mathrm{ml}$ DAPI (Thermo Fischer Scientific, D1306) was used to co-stain the nuclei. IF stained sections were imaged using a Nikon A1 confocal microscope.

mESC culture and the generation Tet-TKO mESCs. mESCs (E14) were cultured on MEFs in Knock-out Dulbecco's Modified Eagle's Medium (Gibco), supplemented with $15 \%$ fetal bovine serum (Omega), $0.5 \%$ penicillin-streptomycin (Gibco), $0.1 \mathrm{mM}$ non-essential amino acids (Gibco), $0.1 \mathrm{mM}$ 2-mercaptoetanol (Sigma), and $103 \mathrm{U} / \mathrm{mL}$ of leukemia inhibitory factor (Millipore). Tet $1 / 2 / 3$ triple knock-out mESCs were generated by the CRISPR/Cas 9 technology as described previously ${ }^{44}$ with slight modifications. Tet1/2/3 sgRNAs were cloned into PX458 (Addgene 48138). Three sgRNAs were transfected simultaneously into mESCs using the iMfectin DNA transfection reagent (Gendepot). mESCs transfected with the vector PX458 without sgRNA were used as control. GFP positive cells were sorted into 96-well plates by flow cytometry and individual colonies were genotyped after 7-day culture.

RFLP analysis and amplicon sequencing. RFLP analysis was performed as described previously ${ }^{44}$. sgRNAs (Supplementary Table 2) targeted to regions of Tet $1 / 2 / 3$ were amplified by PCR and $10 \mu \mathrm{l}$ of products were digested with SacI EcoRV or Xhol, respectively. Digested DNA was separated on 2\% SYBR Safe (Gendepot) stained agarose gel. For amplicon sequencing, PCR products were purified using MinElute PCR purification kit (Qiagen) and libraries generated using Nextera XT DNA library prep kit (Illumina) according to their manuals. Libraries were sequenced on Illumina NextSeq 500 system using the NextSeq 500 High Output v2 Kit (Illumina, San Diego, CA) with a customized single end, single indexing (80/8-bp) format.

Nuclear fractionation and western blot. Nuclear fractionation in mESCs was performed as described previously ${ }^{60}$. Briefly, 10 million cells were washed by PBS and pelleted at $200 \mathrm{~g}$ for $2 \mathrm{~min}$. $200 \mu \mathrm{l}$ buffer A (10 mM HEPES, pH 7.9, $10 \mathrm{mM}$ $\mathrm{KCl}, 1.5 \mathrm{mM} \mathrm{MgCl}_{2}, 0.34 \mathrm{M}$ Sucrose, $10 \%$ Glycerol, $0.1 \%$ Triton X-100, $1 \mathrm{mM}$ DTT, and protease inhibitor cocktail) was added to the cell pellets and incubated on ice for $8 \mathrm{~min}$ to remove the cytoplasm. After centrifugation at $1300 \mathrm{~g}, 4^{\circ} \mathrm{C}$, for 5 min, $100 \mu \mathrm{l}$ Buffer N (15 mM Tris- $\mathrm{HCl}$ [pH 7.5], $200 \mathrm{mM} \mathrm{NaCl}, 60 \mathrm{mM} \mathrm{KCl}, 5 \mathrm{mM}$ $\mathrm{MgCl}_{2}, 1 \mathrm{mM} \mathrm{CaCl}_{2}, 0.3 \% \mathrm{NP}-40$, and protease inhibitor cocktail) was added to the nuclear pellets and incubated on ice for $30 \mathrm{~min}$ to lyse. After centrifugation at 1700 $\mathrm{g}, 4^{\circ} \mathrm{C}$, for $5 \mathrm{~min}$, the supernatant was collected and labeled as soluble fraction, and $100 \mu \mathrm{l}$ sample loading buffer was added to the chromatin pellets for denaturing. Denatured proteins were loaded to the $4-12 \%$ gradient SDS-PAGE (GenScript). Nitrocellulose membranes (Millipore) were used for transferring after gel running. After blocking in 5\% non-fat milk, the membranes were probed with the corresponding primary antibodies overnight at $4{ }^{\circ} \mathrm{C}$, followed by incubation with a secondary antibody at room temperature for $1 \mathrm{~h}$. After adding the West-Q Pico Dura ECL Solution (Gendeport), the antigen-antibody complexes were detected by the ChemiDoc Imaging system (Bio-Rad). The intensity of protein bands was measured by the Image Lab software package (Bio-Rad). The uncropped and unprocessed scans of the blots are available in source data file.

Expression of FLAG-tagged Tet1CD. FLAG-tagged Tet1 catalytic domain (FLAG Tet1CD) was amplified from Fuw-dCas9-Tet1CD (Addgene \#84475) using the primers listed in Supplementary Table 2, and then inserted into a lentiviral vector, 213-PRRL-CAG-NLS-sfGFP (a gift from Dr. Courtney Hodge at Baylor College of Medicine) between the restriction sites NheI (NEB R0131s) and EcoRV (NEB R0195S). The lentiviral vector encoding FLAG-Tet1CD plasmid was transfected into HEK 293 T cells, along with standard virus packaging vectors psPAX2 (Addgene \#12260) and Pmd2.G (Addgene \#12259). The virus-containing supernatants were collected 2 days after transfection followed by brief centrifugation $\left(750 \mathrm{~g}\right.$ at $4^{\circ} \mathrm{C}$ for $\left.10 \mathrm{~min}\right)$. The supernatant with packaged viruses were added into Tet TKO mESCs followed by centrifugation $\left(750 \mathrm{~g}\right.$ at $37^{\circ} \mathrm{C}$ for $\left.90 \mathrm{~min}\right)$. Tet1CD 
expression and subsequent DNA hydroxymethylation was confirmed by Western blotting and a $5 \mathrm{hmC}$ dot blot assay, respectively, 2 days after lentivirus transduction.

Nucleic acid isolation. Total genomic DNA and RNA samples were isolated using the AllPrep DNA/RNA Mini Kit (Qiagen) according to the manufacturer's instructions. In brief, $600 \mu \mathrm{l}$ of buffer RLT plus was added to the embryonic heart tissues or cell pellets. In total $26 \mathrm{G}$ needles (BD) were used to disrupt samples. Lysate was transferred to an AllPrep DNA spin column and centrifuged at $10,000 \times g$ for $30 \mathrm{~s}$. The genomic DNA was captured by the column and $100 \mu \mathrm{l}$ elution buffer was added to elute genomic DNA. Total RNA was in the flowthrough and precipitated by one volume of $70 \%$ ethanol. Then the mixture was transferred to a RNeasy spin column. Purified total RNA was eventually eluted by $30 \mu \mathrm{l}$ RNase-free water. DNA and RNA concentrations were measured by the Qubit fluorometer (Thermo Fisher Scientific).

Dot-blot assay. Purified genomic DNA was denatured in $0.4 \mathrm{M} \mathrm{NaOH}, 10 \mathrm{mM}$ EDTA at $95^{\circ} \mathrm{C}$ for $10 \mathrm{~min}$, then neutralized with ice-cold $2 \mathrm{M}$ ammonium acetate (pH 7.0). Two-fold serial dilutions of the denatured DNA samples were generated and spotted on a nitrocellulose membrane by using an assembled Bio-Dot apparatus (Bio-Rad) according to the manufacturer's instructions. A synthetic oligonucleotide with a known amount of $5 \mathrm{hmC}$ was used as standard ${ }^{1}$. The membrane was washed with $2 \mathrm{xSSC}$ buffer briefly, air-dried and vacuum-baked at $80^{\circ} \mathrm{C}$ for $2 \mathrm{~h}$. DNA hybridized membrane was blocked with $5 \%$ non-fat milk for $1 \mathrm{~h}$ at room temperature and incubated with an anti-5hmC antibody (1:3000, Active Motif) overnight at $4{ }^{\circ} \mathrm{C}$. Next day, the membrane was incubated with a horseradish peroxidase-conjugated anti-rabbit IgG secondary antibody (1:10,000; Sigma) for 1 $\mathrm{h}$ at room temperature. The membrane was visualized by West-Q Pico Dura ECL Solution (GenDEPOT). The membrane was washed with $1 \times$ TBST briefly and then stained with $0.02 \%$ methylene blue in $0.3 \mathrm{M}$ sodium acetate ( $\mathrm{pH} 5.2$ ) to confirm the total amounts of loaded DNA samples. The uncropped and unprocessed scans of the blots are available in source data file.

Real-time quantitative PCR (qPCR). Purified total RNA (10 pg to $5 \mu \mathrm{g}$ ) was reverse transcribed into cDNA with the amfiRivert cDNA Synthesis Platinum Master Mix (Gendepot). Real-time quantitative PCR was performed with a LightCycle 96 (Roche) instrument using amfiSure qGreen Q-PCR Master Mix (Gendepot). Three-step cycling program was used with $3 \mathrm{~min} 95^{\circ} \mathrm{C}$ initial denaturation and $40 \mathrm{cycles}$ of $10 \mathrm{~s} 95^{\circ} \mathrm{C}$ denaturation, $20 \mathrm{~s} 60^{\circ} \mathrm{C}$ annealing and $30 \mathrm{~s} 72^{\circ}$ $\mathrm{C}$ extension. All the primers were synthesized from Integrated DNA Technologies and listed in Supplementary Table 2.

RNA-seq library construction and data analysis. Poly-A tailed messenger RNA was enriched with a Poly(A)Purist ${ }^{\mathrm{m}} \mathrm{MAG}$ Kit (Thermo Fisher Scientific). Enriched mRNA was used for RNA-seq library preparation by using a NEBNext ${ }^{\circledast}$ Ultra $^{\text {Tx }}$ Directional RNA Library Prep Kit (NEB) according to the manufacturer's instructions. The quality of libraries was checked by an Agilent High Sensitivity DNA kit (Agilent Technologies). The library was sequenced using an Illumina NextSeq 500 instrument (150 cycle, paired-end; Supplementary Table 1).

RNA-seq data were mapped to mm10 genome assembly using tophat-2.1.1 with default parameters. Cufflinks and cuffdiff were used to call significantly differentially expressed genes (DEGs) (q-value $<=0.05$ ) between WT and Tet2/3DKO groups at E12.5 and E15.5 developmental stages. In-house R scripts were used to plot the scatter plot for DEGs. DEGs functional enrichment was performed using GSEA ${ }^{61}$. RNA-seq data of mouse embryo hearts ranging from E10.5 to P0 were downloaded from ENCODE (https://www.encodeproject.org/). R package gplots was used to plot heatmaps for DEGs.

scRNA-seq library preparation and data analysis. Embryonic hearts were harvested and digested into single cells using $1 \mathrm{mg} / \mathrm{ml}$ collagenase I (Worthington). Single-cell RNA-seq libraries were generated using the Chromium Single-Cell $3^{\prime}$ Reagent V2 Kit ( $10 \times$ Genomics) according to the manufacturer's protocol. Briefly, single cell GEM was generated and barcoded in a Chromium Controller (10x Genomics). Then RNA transcripts from single cells were reverse transcribed, amplified and fragmented. Library generation was finished by incorporating the adapter and sample indices into the fragmented cDNA. Agilent Bioanalyzer 2100 (Agilent) was used to profile the sizes of the pre-amplified cDNA and the libraries. Libraries were subjected to highthroughput sequencing on a Illumina NextSeq 500 system using the NextSeq 500 High Output v2 Kit (Illumina) with a customized paired end, dual indexing (26/8/0/58-bp) format as recommended by $10 \times$ Genomics.

Cellranger (10XGenomics) was used to generate fasta files and count reads on each gene for each cell. Cellranger count output files were taken as input for $\mathrm{R}$ package seruat to perform single cell analysis. We first used illumina bcl2fastq v2.20.0.422 to demultiplex the raw sequencing data. Cellranger v2.1.1 (https:// support.10xgenomics.com/single-cell-gene-expression/software/pipelines/latest/whatis-cell-ranger) was used to align raw fastq files to $\mathrm{mm} 10$ and perform barcode counting and UMI counting. The count matrix (column as cell; row as genes) from Cellranger count were taken as input for $\mathrm{R}$ package seruat $\mathrm{v} 2.3$ to perform single cell analysis. Reads with the same UMI were combined and then annotated to ensemble genes (GRCm38/mm10). We filtered out the cells with $<100$ genes expressed and keep the cells with $<15 \%$ mitochondria reads rate. The default setting of Seurat v2.362 was used to perform PCA. We used the first 20 principle components to perform cell cluster and t-SNE (resolution =0.6). Markov Affinity-based Graph Imputation of Cells (MAGIC) ${ }^{39}$ was used to perform the PCA and gene interaction analysis as guided by https://github.com/KrishnaswamyLab/MAGIC.

WGBS library construction and data analysis. Purified genomic DNA (with $5 \%$ of unmethylated lambda DNA spike-in, Promega) was sheared to till reaching a fragment size of 200-500 bp using Bioruptor UCD300 (Diagenode) according to manufacturer's instructions. Sheared DNA was ligated with methylated adaptors $\left(\right.$ NEBNext $^{\circledR}$ Multiplex Oligos for Illumina $\left.{ }^{\circledR}, \mathrm{NEB}\right)$ by using a NEBNext ${ }^{\circledR}$ Ultra $^{\text {mm }}$ II DNA Library Prep Kit (NEB). Methylated adaptor-ligated DNA fragment was used for bisulfited conversion reaction with EZ DNA Methylation-Lightning Kit (Zymo Research), then bisulfite converted DNA was amplified using KAPA HiFi HotStart Uracil + ReadyMix PCR Kit (Kapa Biosystems) with 8 cycles of PCR. Amplified DNA was purified by AMPureXP beads and examined by Agilent High Sensitivity DNA kit (Agilent Technologies) for quality check. Library concentration was determined by a Qubit 4 fluorometer (Thermo Fisher Scientific). Prepared libraries were sequenced using an Illumina NextSeq 500 instrument (150-cycle, paired-end).

Raw fastq files for WGBS (from E10.5-P0 stage) were downloaded from ENCODE. Raw fastq files were mapped to the hg19/mm10 genome assembly using bsmap-2.89 software with "-v 6 -n 1 -q 3 -r 1" parameters. The bisulfite conversion ratios were estimated using unmethylated lambda DNA. Mcall modual in $\mathrm{MOABS}^{63}$ was used to call the mCG/CG ratios for each CpG site. Mcomp modual was used to call DMRs with parameter "-minNominalDif $=0.2-\operatorname{minDmcsInDmr}$ 3-maxDistConsDmcs 500". The CpGs with coverage $>=5$ was used for downstream analysis. The function prediction of DMRs was used for GREAT analysis $^{26}$. UCSC genome browser tracks were generated by using the Mmint ucsc. py function.

CMS-IP-seq library construction and data analysis. CMS-IP-seq were performed as described previously with some modification ${ }^{40,41}$. Purified genomic DNA was sheared to yield 200-500 bp fragments using Bioruptor UCD300 (Diagenode) according to manufacturer's instructions. Bisulfite conversion was performed using the fragmented DNA with an EZ DNA Methylation-Lightning Kit (Zymo Research) to convert $5 \mathrm{hmC}$ to cytosine methyl sulfonate (CMS). CMScontaining DNA fragments were enriched using anti-CMS antibody and protein A/ $\mathrm{G}$ dynabeads (Thermo Fisher Scientific). Enriched DNA fragments were then purified using the well-established phenol-chloroform-isoamyl alcohol extraction method. Purified DNA was then processed with a Pico Methyl-Seq Library Prep Kit (Zymo Research) to generate sequencing libraries. The quality of DNA libraries was checked by an Agilent High Sensitivity DNA kit (Agilent Technologies), and then subjected to highthroughput sequencing on an Illumina NextSeq 500 instrument (75-cycle, single-end) (Supplementary Table 1).

Raw fastq data was mapped to the mm10/hg19 genome assembly using bsmap2.89 with default parameters. After duplication removal, CMS peaks were called by using macs 2 with default parameters. Bedtools merge was used to generate merged peaks for all samples. Reads numbers in each peak were counted if there is $>1 \mathrm{bp}$ overlap between reads location and peak region. The raw counts file with row as each peak, column as samples was used as input to DEGseq2 and differentially significantly CMS peaks ( $\mathrm{q}$ value $<=0.05$ ) between WT and Tet $2 / 3-\mathrm{DKO}$ were called. Volcano plots were plotted using $\mathrm{R}$ package ggplot2. The functions of decrease/increased CMS peaks between WT and DKO were predicted by using GREAT analysis ${ }^{26}$. Histone peaks regions and fold change over control bigwig files were downloaded from ENCODE (mm10 version). The average fold change of histone modifications signals over control on HypoDHMRs/HyperDHMRs and the average signal of CMS-IP-seq along metagenes were used with Mmint (https://github.com/lijiacd985/Mmint).

ATAC-seq library construction and data analysis. ATAC-seq library preparation was performed, as described previously ${ }^{64}$. Briefly, 50,000 cells were collected in ice-cold PBS. Nuclei were isolated in Cold Lysis Buffer $(10 \mathrm{mM}$ Tris- $\mathrm{HCl}, \mathrm{pH} 7.4$, $10 \mathrm{mM} \mathrm{NaCl}, 3 \mathrm{mM} \mathrm{MgCl} 2,0.1 \%$ IGEPAL). The transposition reaction was performed by using a Nextera DNA Library Preparation Kit (Illumina) with modified tagmentation condition $\left(37^{\circ} \mathrm{C}\right.$ for $\left.30 \mathrm{~min}\right)$. Tagmented DNA was purified by E.Z. N.A. ${ }^{\circledR}$ MicroElute Cycle Pure Kit (Omega BIO-TEK), then amplified with the KAPA real-time library amplification kit (Kapa Biosystems) followed by library purification using AmpuXP beads. The quality of purified DNA libraries was checked by Agilent High Sensitivity DNA kit (Agilent Technologies). The library was sequenced using an Illumina NextSeq 500 instrument (150 cycle, paired-end) (Supplementary Table 1).

Bowtie 2 with '-very-sensitive' option was used to map the high-quality reads to mml0 version of human genome. The uniquely properly paired mapped reads were extracted for downstream analysis. MACS2 with the '-nomodel' and '-extsize $147^{\prime}$ was used to call ATAC peaks. Bedtools intersect (at least 1 bp overlap) was used to identify overlapped $5 \mathrm{hmC}$ peaks and ATAC peaks. We first cut the genome 
to $10 \mathrm{~kb}$ equal size bins and use bigwigOverbed to calculate each bin's $5 \mathrm{hmC}$ and ATAC signals. R package geneplotter was used to plot the density scatterplots. The findMotifsGenome.pl in HOMER software was used for the motif enrichment with default setting. Randomly selected sequences from the genome with matched GC\% content were used as background.

Cleavage under targets and release using nuclease (Cut\&Run). Cut\&Run was performed according to published protocol ${ }^{65}$. Briefly, single cells were attached to the concanavalin A-coated magnetic beads (Bangs Laboratories) followed by the in situ binding of the antibody and pA-MN specifically to the target protein. Cleaved fragments were released after exposure to calcium. DNA was extracted from the supernatant containing released chromatin fragments. Libraries were prepared using the ThruPLEX DNA-seq Kit from Rubicon Genomics (R400406) according to the manufacture's instruction with slightly modification of changing extension time of library amplification steps to $20 \mathrm{~s}$. Libraries were sequenced using the NextSeq 500 High Output v2 Kit (Illumina, San Diego, CA) with a customized paired end, dual indexing (40/8/0/40-bp) format (Supplementary Table 1).

We analyzed CUT\&RUN data using the script on github (https://github.com/ Henikoff/Cut-and-Run). Briefly, we first mapped paired end raw fastq files to $\mathrm{mm} 10$ use bowtie 2.2.5 with parameters "-local-very-sensitive-local-no-unal-nomixed-no-discordant-phred33 -I 10 -X 700". Then we used picard to remove duplication reads; bamToBed was used to transform bam file to bed file. Next, we used spike_in_calibration.csh script to perform spike in normalization. BedGraphtobigwig was used to transform the bedGraph file to bigwig file, which was used to perform the visualization.

HiChIP library construction and data analysis. HiChIP library preparation was performed as described previously ${ }^{49}$. Briefly, 1 million crosslinked cardiac cells from mouse embryos or 2 million mouse embryonic stem cells were lysed with $\mathrm{Hi}$ C Lysis Buffer (10 mM Tris-HCl pH 8.0, $10 \mathrm{mM} \mathrm{NaCl}, 0.2 \%$ NP- 40 with $1 \times$ protease inhibitor cocktail), followed by digestion with $150 \mathrm{U}$ of MboI restriction enzyme (NEB) for $2 \mathrm{~h}$ at $37^{\circ} \mathrm{C}$. Fill-in master mix containing biotin-dATP (Thermo Fisher Scientific) was added to digest nuclei to generate enzyme-digested overhang and mark the DNA ends with biotin. Subsequently, T4 DNA ligase (NEB) was added to the reaction and incubated for $4 \mathrm{~h}$ at room temperature to achieve proximity ligation. After that, nuclei were resuspended with Nuclear Lysis Buffer (50 mM Tris-HCl pH7.5, $10 \mathrm{mM} \mathrm{EDTA,} 1 \%$ SDS with $1 \times$ protease inhibitor cocktail) and transferred to Covaris millitube for fragmentation. Fragmented samples were precleared by adding protein A/G beads (Thermo Fischer Scientific) for $1 \mathrm{~h}$ at $4{ }^{\circ} \mathrm{C}$, and $2 \mu \mathrm{g}$ Smcla antibody was added with an overnight incubation. On day 2, protein A beads (Thermo Fisher Scientific) were added to the reaction for $2 \mathrm{~h}$ to capture the beads. After bead capturing, the samples were washed three times each with low salt wash buffer $(0.1 \%$ SDS, $1 \%$ Triton X-100, 2 mM EDTA, 20 $\mathrm{mM}$ Tris-HCl pH 7.5, $150 \mathrm{mM} \mathrm{NaCl})$, high salt wash buffer $(0.1 \%$ SDS, $1 \%$ Triton $\mathrm{X}-100,2 \mathrm{mM}$ EDTA, $20 \mathrm{mM}$ Tris-HCl pH 7.5, $500 \mathrm{mM} \mathrm{NaCl}$ ), and $\mathrm{LiCl}$ wash buffer $(10 \mathrm{mM}$ Tris-HCl pH 7.5, $250 \mathrm{mM} \mathrm{LiCl,} 1 \% \mathrm{NP}-40,1 \%$ sodium deoxycholate, $1 \mathrm{mM}$ EDTA) at room temperature. After these steps, ChIP samples were resuspended in a DNA elution buffer (50 $\mathrm{mM}$ sodium bicarbonate pH8.0, $1 \% \mathrm{SDS}$ ) and incubated for $10 \mathrm{~min}$ at room temperature, followed by shaking for $3 \mathrm{~min}$ at $37^{\circ} \mathrm{C}$. DNA eluted from the beads were collected twice, followed by reverse crosslinking. Reverse crosslinked DNA were then purified by using an E.Z.N.A. ${ }^{\oplus}$ MicroElute Cycle Pure Kit (Omega BIO-TEK). Purified samples were used for biotin pull-down. Resuspended Streptavidin C-1 (Thermo Fisher Scientific) with $2 \times$ Biotin binding buffer $(10 \mathrm{mM}$ Tris- $\mathrm{HCl}$ pH7.5, $1 \mathrm{mM}$ EDTA, $2 \mathrm{M} \mathrm{NaCl})$ was added to the samples and proceed biotin capturing procedure by incubating $15 \mathrm{~min}$ at room temperature, followed by washes with Tween wash buffer $(5 \mathrm{mM}$ Tris- $\mathrm{HCl}$ pH7.5, $0.5 \mathrm{mM}$ EDTA, $1 \mathrm{M} \mathrm{NaCl}, 0.05 \%$ Tween-20) and $1 \times$ TD buffer $(10 \mathrm{mM}$ Tris- $\mathrm{HCl} \mathrm{pH} 7.5,5 \mathrm{mM}$ magnesium chloride, $10 \%$ demethylformamide), respectively. After wash, on-bead tagmentation by using $\mathrm{Tn} 5$ transposase (Illumina) was performed for $10 \mathrm{~min}$ at $55^{\circ} \mathrm{C}$ with interval shaking, followed by several washes with $50 \mathrm{mM}$ EDTA, Tween-20 wash buffer, and $10 \mathrm{mM}$ Tris- $\mathrm{HCl}$ respectively. After wash, the reaction beads were resuspended in a PCR master mix $\left(\mathrm{Q}^{\circledR}{ }^{\circledR} \mathrm{High}-\right.$ Fidelity $2 \times$ Master Mix, NEB, with Nextera Ad1.1 (Universal) and Ad2.X (barcoded) primers) for library amplification. Amplified on-bead DNA were eluted using a magnet and purified with an E.Z.N.A. ${ }^{\oplus}$ MicroElute Cycle Pure Kit (Omega BIO-TEK). The quality of libraries was checked by Agilent High Sensitivity DNA kit (Agilent Technologies). The library was sequenced on an Illumina NextSeq 500 instrument (150 cycle, paired-end) (Supplementary Table 1).

A total of 1 million cells from each condition were used to perform HiCHIP experiments. To improve the statistic power, we merged the two biological replicates to increase sequencing depth. HiC-Pro ${ }^{66}$ was used to map the raw paired-end fastq files to mm10 genome assembly and identify the uniquely validated paired reads. build_raw_maps.sh and ice_norm.sh embeded in HiC-Pro pipeline were used to generate the raw contact map and normalized contact map. Fit-Hi-C ${ }^{50}$ was used to identify the significant Paired End Tags (PETs) between any two bins $(5 \mathrm{~kb})$ with a $p$ value $<0.05$. We linked the PETs with the whole genic regions. The PETs linked to specific genes were counted and normalized, as shown in a previous study ${ }^{49}$. The RNA-seq, CMS-IP, ATAC-seq, ChIP-seq data and pairwise files containing PETs information were uploaded to WashU Epigenome Browser $^{67}$ (http://epigenomegateway.wustl.edu).
Prediction of AB compartment. The output file *_allValidPairs.hic from HiC-Pro pipeline was used as input file for juicer_tools.1.7.5_linux_x64_jcuda.0.8.jar eigenvector function ${ }^{68}$. The ${ }^{*}$ allValidPairs.hic file stores all the raw interaction paired reads between any two genomic bins from the same chromosome. The eigenvector for each chromosome with KR normalization at $50 \mathrm{~kb}$ resolution were calculated. For each chromosome, we manually checked the overlap between the compartment assignment and the accessible regions from our ATAC-seq data to decide if the compartment assignment need to be flipped.

\section{Chromatin immunoprecipitation quantitative PCR (ChIP-qPCR). ChIP was} performed according to a previously described protocol with slight modifications ${ }^{69}$ Briefly, 20 million mESCs were fixed with $1 \%$ formaldehyde for $15 \mathrm{~min}$ at room temperature followed by quenching with $125 \mathrm{mM}$ glycine for $5 \mathrm{~min}$. Cells were washed using ice cold PBS twice and then resuspended in a sonication buffer $(10$ $\mathrm{mM}$ Tris $\mathrm{pH} 8.0,0.25 \% \mathrm{SDS}, 2 \mathrm{mM}$ EDTA and protease inhibitor cocktail). The M220 Focused-ultrasonicator (Covaris) was used to sonicate the chromatin into $200-700$ bp range. Pre-washed $25 \mu \mathrm{l}$ protein G Dynabeads, $10 \mu \mathrm{g}$ YY1 antibody and sheared chromatin were incubated overnight. The enrichment mixture was washed twice with each of the following buffers: RIPA-low salt $(10 \mathrm{mM}$ Tris $\mathrm{HCl} \mathrm{pH} 8.0$, $140 \mathrm{mM} \mathrm{NaCl}, 1 \mathrm{mM}$ EDTA pH 8.0, $0.1 \%$ SDS, $0.1 \% \mathrm{Na}$-Deoxycholate, $1 \%$ Triton X-100 and protease inhibitor cocktail), RIPA-high salt ( $10 \mathrm{mM}$ Tris $\mathrm{HCl} \mathrm{pH} \mathrm{8.0,}$ $500 \mathrm{mM} \mathrm{NaCl}, 1 \mathrm{mM}$ EDTA pH 8.0, 0.1\% SDS, $0.1 \%$ Na-Deoxycholate, $1 \%$ Triton $\mathrm{X}-100$ and protease inhibitor cocktail), RIPA-LiCl (10 mM Tris $\mathrm{HCl} \mathrm{pH} \mathrm{8.0,}$ $250 \mathrm{mM} \mathrm{LiCl}, 1 \mathrm{mM}$ EDTA pH 8.0, 0.1\% SDS, 0.1\% Na-Deoxycholate, 0.5\% NP-40 and protease inhibitor cocktail) and TE buffer $(10 \mathrm{mM}$ Tris $\mathrm{HCl}$ pH8.0, $1 \mathrm{mM}$ EDTA pH 8.0 and protease inhibitor cocktail). After). IP fragments were incubated at $55^{\circ} \mathrm{C}$ for $1 \mathrm{~h}$ in elution buffer $(10 \mathrm{mM}$ Tris $\mathrm{HCl}$ pH8.0, $5 \mathrm{mM}$ EDTA pH 8.0, $300 \mathrm{mM} \mathrm{NaCl}, 0.4 \% \mathrm{SDS}$ ). The elution were reverse crosslinked at $65^{\circ} \mathrm{C}$ overnight with $2 \mu \mathrm{l} 20 \mathrm{mg} / \mathrm{ml}$ proteinase $\mathrm{K}$. DNA fragments were purified using MicroElute Cycle-Pure Kit (OMEGA). qPCR was performed following the protocol described above.

4C-seq library construction and data analysis. 4C-seq was performed as previously described ${ }^{47}$. In total 10 million mESCs were crosslinked with $1 \%$ formaldehyde in PBS contains 10\% FBS for 10 min. Glycine was added to a final concentration of $125 \mathrm{mM}$ to quench the reaction. Cells were then washed twice using ice cold PBS followed by snap freezing with liquid nitrogen and stored at $-80^{\circ} \mathrm{C}$. Ice cold Hi-C lysis buffer $(10 \mathrm{mM}$ Tris- $\mathrm{HCl} \mathrm{pH8.0,10} \mathrm{mM} \mathrm{NaCl}, 0.2 \%$ Igepal and protease inhibitor cocktail) was used to isolate the nuclei. For the Mpdu1 loci, DpnII (NEB R0543) was used for the primary digestion and BfaI (NEB R0568S) was used for the secondary digestion. PCR was performed using the Roche Expand Long Template polymerase (Roche 11759060001). Libraries were generated using the the NEB Next Ultra II DNA Library Prep Kit prior to sequencing (NEB \#E7103). All the oligonucleotides are listed in Supplementary Table 2. Basic4Cseq R package was used to calculate normalized $4 \mathrm{C}$ signals and plot $4 \mathrm{C}$ signals nearby the targeted regions.

Reporting summary. Further information on research design is available in the Nature Research Reporting Summary linked to this article.

\section{Data availability}

The WGBS, CMS-IP, ATAC-seq, CUT\&RUN, and RNA-seq (bulk and single-cell) data from this study have been submitted to the NCBI Gene Expression Omnibus (GEO; https://www.ncbi.nlm.nih.gov/geo/) under accession number GSE121671. All relevan data supporting the key findings of this study are available within the article and its Supplementary Information files or from the corresponding author upon reasonable request. The source data underlying Figs 1e, 2b-d, 4a, 6a, 7h and Supplementary Figs $3 \mathrm{f}$ and $8 \mathrm{a}$ are provided as a Source Data file. A reporting summary for this Article is available as a Supplementary Information file.

Received: 1 October 2018 Accepted: 30 August 2019

Published online: 20 September 2019

\section{References}

1. Kang, J. et al. Simultaneous deletion of the methylcytosine oxidases Tet 1 and Tet3 increases transcriptome variability in early embryogenesis. Proc. Natl Acad. Sci. USA 112, E4236-E4245 (2015)

2. Dai, H. Q. et al. TET-mediated DNA demethylation controls gastrulation by regulating Lefty-Nodal signalling. Nature 538, 528-532 (2016).

3. $\mathrm{Gu}, \mathrm{T}$. P. et al. The role of Tet3 DNA dioxygenase in epigenetic reprogramming by oocytes. Nature 477, 606-610 (2011).

4. Dawlaty, M. M. et al. Loss of tet enzymes compromises proper differentiation of embryonic stem cells. Dev. Cell 29, 102-111 (2014). 
5. Verma, N. et al. TET proteins safeguard bivalent promoters from de novo methylation in human embryonic stem cells. Nat. Genet. 50, 83-95 (2018).

6. Tahiliani, M. et al. Conversion of 5-methylcytosine to 5-hydroxymethylcytosine in mammalian DNA by MLL partner TET1. Science 324, 930-935 (2009).

7. He, Y. F. et al. Tet-mediated formation of 5-carboxylcytosine and its excision by TDG in mammalian DNA. Science 333, 1303-1307 (2011).

8. Ito, $\mathrm{S}$. et al. Tet proteins can convert 5-methylcytosine to 5-formylcytosine and 5-carboxylcytosine. Science 333, 1300-1303 (2011).

9. Ko, M. et al. Impaired hydroxylation of 5-methylcytosine in myeloid cancers with mutant TET2. Nature 468, 839-843 (2010).

10. Lio, C. W., et al. Tet 2 and Tet 3 cooperate with B-lineage transcription factors to regulate DNA modification and chromatin accessibility. Elife 5, e18290 (2016).

11. Bogdanovic, O. et al. Active DNA demethylation at enhancers during the vertebrate phylotypic period. Nat. Genet 48, 417-426 (2016).

12. Lee, M. et al. Engineered Split-TET2 Enzyme for Inducible Epigenetic Remodeling. J. Am. Chem. Soc. 139, 4659-4662 (2017).

13. Li, J., et al. Decoding the dynamic DNA methylation and hydroxymethylation landscapes in endodermal lineage intermediates during pancreatic differentiation of hESC. Nucleic Acids Res. 46, 2883-2900 (2018).

14. Jaiswal, S. et al. Age-related clonal hematopoiesis associated with adverse outcomes. N. Engl. J. Med. 371, 2488-2498 (2014).

15. Fuster, J. J. et al. Clonal hematopoiesis associated with TET2 deficiency accelerates atherosclerosis development in mice. Science 355, 842-847 (2017).

16. Sano, S. et al. Tet2-mediated clonal hematopoiesis accelerates heart failure through a mechanism involving the IL-1beta/NLRP3 inflammasome. J. Am. Coll. Cardiol. 71, 875-886 (2018).

17. Paige, S. L. et al. Molecular regulation of cardiomyocyte differentiation. Circ. Res. 116, 341-353 (2015).

18. Gilsbach, R. et al. Dynamic DNA methylation orchestrates cardiomyocyte development, maturation and disease. Nat. Commun. 5, 5288 (2014).

19. Gilsbach, R. et al. Distinct epigenetic programs regulate cardiac myocyte development and disease in the human heart in vivo. Nat. Commun. 9, 39 (2018).

20. Greco, C. M. et al. DNA hydroxymethylation controls cardiomyocyte gene expression in development and hypertrophy. Nat. Commun. 7, 12418 (2016).

21. Serra-Juhe, C. et al. DNA methylation abnormalities in congenital heart disease. Epigenetics 10, 167-177 (2015)

22. Li, D. \& Rozen, R. Maternal folate deficiency affects proliferation, but not apoptosis, in embryonic mouse heart. J. Nutr. 136, 1774-1778 (2006).

23. Watkins, M. L. \& Botto, L. D. Maternal prepregnancy weight and congenital heart defects in offspring. Epidemiology 12, 439-446 (2001).

24. Krishnan, A. et al. A detailed comparison of mouse and human cardiac development. Pediatr. Res. 76, 500-507 (2014).

25. Yue, F. et al. A comparative encyclopedia of DNA elements in the mouse genome. Nature 515, 355-364 (2014).

26. McLean, C. Y. et al. GREAT improves functional interpretation of cisregulatory regions. Nat. Biotechnol. 28, 495-501 (2010)

27. Ko, M. et al. Modulation of TET2 expression and 5-methylcytosine oxidation by the CXXC domain protein IDAX. Nature 497, 122-126 (2013).

28. Huang, Y. et al. The behaviour of 5-hydroxymethylcytosine in bisulfite sequencing. PLoS ONE 5, e8888 (2010).

29. Inoue, A. \& Zhang, Y. Replication-dependent loss of 5-hydroxymethylcytosine in mouse preimplantation embryos. Science 334, 194 (2011).

30. Dawlaty, M. M. et al. Tet1 is dispensable for maintaining pluripotency and its loss is compatible with embryonic and postnatal development. Cell Stem Cell 9, 166-175 (2011)

31. Quivoron, C. et al. TET2 inactivation results in pleiotropic hematopoietic abnormalities in mouse and is a recurrent event during human lymphomagenesis. Cancer Cell 20, 25-38 (2011).

32. Moran-Crusio, K. et al. Tet2 loss leads to increased hematopoietic stem cell self-renewal and myeloid transformation. Cancer Cell 20, 11-24 (2011).

33. Ko, M. et al. Ten-Eleven-Translocation 2 (TET2) negatively regulates homeostasis and differentiation of hematopoietic stem cells in mice. Proc. Natl Acad. Sci. USA 108, 14566-14571 (2011).

34. Li, Z., et al. Deletion of Tet2 in mice leads to dysregulated hematopoietic stem cells and subsequent development of myeloid malignancies. Blood 118 4509-4518 (2011).

35. Moses, K. A. et al. Embryonic expression of an Nkx2-5/Cre gene using ROSA26 reporter mice. Genesis 31, 176-180 (2001).

36. Wilsbacher, L. \& McNally, E. M. Genetics of cardiac developmental disorders: cardiomyocyte proliferation and growth and relevance to heart failure. Annu Rev. Pathol. 11, 395-419 (2016).

37. Luxan, G. et al. Mutations in the NOTCH pathway regulator MIB1 cause left ventricular noncompaction cardiomyopathy. Nat. Med. 19, 193-201 (2013).

38. D'Amato, G. et al. Sequential Notch activation regulates ventricular chamber development. Nat. Cell Biol. 18, 7-20 (2016).
39. van Dijk, D. et al. Recovering gene interactions from single-cell data using data diffusion. Cell 174, 716-729 e727 (2018)

40. Huang, Y. et al. The anti-CMS technique for genome-wide mapping of 5hydroxymethylcytosine. Nat. Protoc. 7, 1897-1908 (2012).

41. Pastor, W. A. et al. Genome-wide mapping of 5-hydroxymethylcytosine in embryonic stem cells. Nature 473, 394-397 (2011).

42. Zhang, X. et al. DNMT3A and TET2 compete and cooperate to repress lineage-specific transcription factors in hematopoietic stem cells. Nat. Genet. 48, 1014-1023 (2016)

43. Gregoire, S. et al. Essential and unexpected role of Yin Yang 1 to promote mesodermal cardiac differentiation. Circ. Res. 112, 900-910 (2013).

44. Wang, H. et al. One-step generation of mice carrying mutations in multiple genes by CRISPR/Cas-mediated genome engineering. Cell 153, 910-918 (2013).

45. Skene, P. J. \& Henikoff, S. An efficient targeted nuclease strategy for highresolution mapping of DNA binding sites. Elife 6, e21856 (2017).

46. Sigova, A. A. et al. Transcription factor trapping by RNA in gene regulatory elements. Science 350, 978-981 (2015)

47. Weintraub, A. S. et al. YY1 is a structural regulator of enhancer-promoter loops. Cell 171, 1573-1588 e1528 (2017).

48. Eagen, K. P. Principles of chromosome architecture revealed by Hi-C. Trends Biochem. Sci. 43, 469-478 (2018).

49. Mumbach, M. R. et al. HiChIP: efficient and sensitive analysis of proteindirected genome architecture. Nat. Methods 13, 919-922 (2016).

50. Ay, F., Bailey, T. L. \& Noble, W. S. Statistical confidence estimation for Hi-C data reveals regulatory chromatin contacts. Genome Res. 24, 999-1011 (2014)

51. Morikawa, Y., Leach, J. \& Martin, J. F. Yin-Yang 1, a new player in early heart development. Circ. Res. 112, 876-877 (2013).

52. Beketaev, I. et al. Critical role of YY1 in cardiac morphogenesis. Dev. Dyn. 244, 669-680 (2015).

53. Gordon, S. et al. Transcription factor YY1: structure, function, and therapeutic implications in cancer biology. Oncogene 25, 1125-1142 (2006).

54. Khoueiry, R. et al. Lineage-specific functions of TET1 in the postimplantation mouse embryo. Nat. Genet. 49, 1061-1072 (2017).

55. Stanley, E. G. et al. Efficient Cre-mediated deletion in cardiac progenitor cells conferred by a 3'UTR-ires-Cre allele of the homeobox gene Nkx2-5. Int J. Dev. Biol. 46, 431-439 (2002).

56. Bauer, C. et al. Phosphorylation of TET proteins is regulated via OGlcNAcylation by the O-linked N-acetylglucosamine transferase (OGT) J. Biol. Chem. 290, 4801-4812 (2015).

57. Carlson, B. Human Embryology and Developmental Biology. (2008).

58. Lu, M. F. et al. Function of Rieger syndrome gene in left-right asymmetry and craniofacial development. Nature 401, 276-278 (1999).

59. Xiao, Y. et al. Hippo signaling plays an essential role in cell state transitions during cardiac fibroblast development. Dev. Cell 45, 153-169 e156 (2018)

60. Veland, N. et al. The arginine methyltransferase PRMT6 regulates DNA methylation and contributes to global DNA hypomethylation in cancer. Cell Rep. 21, 3390-3397 (2017).

61. Subramanian, A. et al. Gene set enrichment analysis: a knowledge-based approach for interpreting genome-wide expression profiles. Proc. Natl Acad. Sci. USA 102, 15545-15550 (2005).

62. Satija, R. et al. Spatial reconstruction of single-cell gene expression data. Nat Biotechnol. 33, 495-502 (2015).

63. Sun, D. et al. MOABS: model based analysis of bisulfite sequencing data Genome Biol. 15, R38 (2014).

64. Buenrostro, J. D. et al. Transposition of native chromatin for fast and sensitive epigenomic profiling of open chromatin, DNA-binding proteins and nucleosome position. Nat. Methods 10, 1213-1218 (2013).

65. Skene, P. J., Henikoff, J. G. \& Henikoff, S. Targeted in situ genome-wide profiling with high efficiency for low cell numbers. Nat. Protoc. 13, 1006-1019 (2018).

66. Servant, N. et al. HiC-Pro: an optimized and flexible pipeline for Hi-C data processing. Genome Biol. 16, 259 (2015).

67. Zhou, X. et al. Exploring long-range genome interactions using the WashU epigenome browser. Nat. Methods 10, 375-376 (2013)

68. Lieberman-Aiden, E. et al. Comprehensive mapping of long-range interactions reveals folding principles of the human genome. Science 326, 289-293 (2009).

69. Schmidl, C. et al. ChIPmentation: fast, robust, low-input ChIP-seq for histones and transcription factors. Nat. Methods 12, 963-965 (2015)

\section{Acknowledgements}

We are grateful for Dr. Jianjun Shen and the MD Anderson Cancer Center nextgeneration sequencing core at Smithville (CPRIT RP120348 and RP170002), and the Epigenetic core in Institute of Biosciences and Technology at the Texas A\&M University This work was supported by grants from Cancer Prevention and Research Institute of Texas (RR140053 to Y.H., RP170660 to Y.Z., RP180131 to D.S.), the Innovation Award from American Heart Association (16IRG27250155 to Y.H.), the John S. Dunn Foundation Collaborative Research Award (to Y.H.), National Institute of Health grants (R01HL134780 to Y.H., R01HL146852 to Y.H., R01DE023177 to J.F.M., R01HL127717 
to J.F.M., R01HL130804 to J.F.M., R01HL118761 to J.F.M., F31HL136065 to M.C.H., R01GM112003 to Y.Z., R01HL123953 to J.C.), the Welch Foundation (BE-191320190330 to Y.Z.), the American Cancer Society (RSG-18-043-01-LIB to YH; RSG-16215-01-TBE to Y.Z.), Vivian L. Smith Foundation and MacDonald Research Fund Award (16RDM001 to J.F.M.), Transatlantic Network of Excellence Award LeDucq Foundation Transatlantic Networks of Excellence in Cardiovascular Research 14CVD01: "Defining genomic topology of atrial fibrillation." (J.F.M.), and by an allocation from the Texas A\&M University start-up funds (Y.H. and D.S.).

\section{Author contributions}

Y.H. and J.F.M. directed and oversaw the project. S.F. and Y.X. performed in vivo embryonic development studies. S.F., W.H., and T.L. maintained the genetically modified mice and performed histological analyses. S.F. and M.L. performed high-throughput sequencing library preparation. S.F. performed the CUT\&RUN, dot-blot, ChIP-qPCR analysis. S.F. and T.H. performed Tet1CD rescue experiment. S.F. and L.G. performed 4C analysis. J.L. and D.S. performed integrative data analysis. R.X. and P.Z. provided human embryo samples. M.H., W.M., F.W., J.C. and Y.Z. provided intellectual inputs. All the authors participated in discussion and data interpretation. Y.H., Y.Z. and J.F.M. wrote the manuscript.

\section{Additional information}

Supplementary Information accompanies this paper at https://doi.org/10.1038/s41467019-12325-Z.

Competing interests: The authors declare no competing interests.
Reprints and permission information is available online at http://npg.nature.com/ reprintsandpermissions/

Peer review information Nature Communications thanks Jonathan Brown, Rajan Jain and the other, anonymous, reviewer(s) for their contribution to the peer review of this work. Peer reviewer reports are available.

Publisher's note Springer Nature remains neutral with regard to jurisdictional claims in published maps and institutional affiliations.

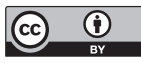

Open Access This article is licensed under a Creative Commons Attribution 4.0 International License, which permits use, sharing, adaptation, distribution and reproduction in any medium or format, as long as you give appropriate credit to the original author(s) and the source, provide a link to the Creative Commons license, and indicate if changes were made. The images or other third party material in this article are included in the article's Creative Commons license, unless indicated otherwise in a credit line to the material. If material is not included in the article's Creative Commons license and your intended use is not permitted by statutory regulation or exceeds the permitted use, you will need to obtain permission directly from the copyright holder. To view a copy of this license, visit http://creativecommons.org/ licenses/by/4.0/.

(C) The Author(s) 2019 